\title{
One-loop Chern-Simons terms in five dimensions
}

\author{
Federico Bonetti, ${ }^{a}$ Thomas W. Grimm ${ }^{a}$ and Stefan Hohenegger ${ }^{b}$ \\ ${ }^{a}$ Max-Planck-Institut für Physik, \\ Föhringer Ring 6, 80805 Munich, Germany \\ ${ }^{b}$ Department of Physics, CERN - Theory Division, \\ CH-1211 Geneva 23, Switzerland \\ E-mail: bonetti@mpp.mpg.de, grimm@mpp.mpg.de, \\ stefan.hohenegger@cern.ch
}

ABSTRACT: We compute one-loop corrections to five-dimensional gauge and gravitational Chern-Simons terms induced by integrating out charged massive fields. The considered massive fields are spin- $1 / 2$ and spin- $3 / 2$ fermions, as well as complex two-forms with first order kinetic terms. Consistency with six-dimensional gravitational anomalies of $(1,0)$ and $(2,0)$ theories is shown by interpreting the massive fields as excited Kaluza-Klein modes in a circle compactification. The results are in accordance with the geometric predictions of the M-theory to F-theory duality as well as the comparison with an explicit one-loop computation in heterotic string theory compactified on $K 3 \times S^{1}$.

Keywords: Chern-Simons Theories, Field Theories in Higher Dimensions, Anomalies in Field and String Theories

ARXIV EPRINT: 1302.2918 


\section{Contents}

1 Introduction $\quad 2$

2 Summary of results 4

3 Field theory computation $\quad 6$

3.1 Minimally coupled massive actions 6

3.2 Computation of the $A \wedge F \wedge F$ coupling 7

3.3 Computation of the $A \wedge \operatorname{tr}(R \wedge R)$ coupling $\quad 10$

3.4 Non-minimal couplings and renormalisation 14

4 Consistency with the M-theory to F-theory limit $\quad 15$

$\begin{array}{lll}4.1 & \text { Field theory prediction } & 15\end{array}$

$\begin{array}{lll}4.2 & \text { F-theory check } & 17\end{array}$

$5 \quad$ Dual heterotic string on $K 3 \times S^{1} \quad 20$

5.1 Heterotic setup 20

$\begin{array}{lll}5.2 & \text { String amplitudes } & 21\end{array}$

$\begin{array}{lll}5.2 .1 & \text { Vertex operators } & 21\end{array}$

5.2.2 World-sheet CFT 21

5.2.3 Explicit amplitudes $\quad 22$

5.3 Change of basis 23

6 Conclusions 24

$\begin{array}{lr}\text { A Notations and conventions } & 26\end{array}$

$\begin{array}{ll}\text { B Gravitational perturbative expansion } & 27\end{array}$

C Feynman rules $\quad 28$

C.1 Spin- $1 / 2$ fermion 28

C.2 Massive self-dual tensor 28

$\begin{array}{lll}\text { C.3 Spin-3/2 fermion } 29 & 29\end{array}$

$\begin{array}{ll}\text { D Torus integration } & 30\end{array}$ 


\section{Introduction}

The derivation of a Wilsonian low-energy effective action amounts to integrating out all excitations beyond a chosen cutoff mass scale and obtaining a theory with modified couplings for the remaining degrees of freedom. The consideration of such theories is crucial, for example, to study physical properties of a fundamental theory such as string theory at low energies. The corrections to the low energy effective action obtained by integrating out massive fields are organised in an expansion in the inverse mass scale. In the limit of large cutoff scale corrections are typically strongly suppressed and can be neglected. In this case all modes with masses above the cutoff scale become effectively non-dynamical and can be decoupled from the theory. This is the subject of well known results in quantum field theory, such as the Appelquist-Carazzone-Symanzik decoupling theorem [1,2]. This reasoning, however, breaks down for certain types of couplings. Four-dimensional examples are furnished by Goldstone-Wilczek currents [3] and Wess-Zumino terms [4] generated by integrating out a fermion that becomes massive via Yukawa coupling to a scalar that gets a non-vanishing VEV. They are independent of the fermion mass and have to be included in the low-energy effective action even in the limit in which it is taken to infinity. In this work we will study couplings with similar features, namely gauge and gravitational Chern-Simons couplings in five-dimensional theories.

The five-dimensional quantum field theories under consideration will propagate both massless and massive degrees of freedom. We will consider massive spin- $1 / 2$ fermions, spin- $3 / 2$ fermions, and complex two-forms. The kinetic and mass terms of the fermions are of standard form while the complex two-forms admit first order kinetic terms. The latter feature is possible in odd-dimensional theories and is crucial for the fields to introduce corrections to Chern-Simons couplings. This can be attributed to the fact that the Chern-Simons couplings violate parity and only fields with parity violating actions can modify their prefactors when deriving the Wilsonian effective action. The massive fields are minimally coupled to a massless $\mathrm{U}(1)$ gauge field $A$ with field strength $F$. We aim to derive the corrections to the gauge Chern-Simons term $A \wedge F \wedge F$ and the gravitational Chern-Simons term $A \wedge \operatorname{tr}(R \wedge R)$, where $R$ is the five-dimensional curvature two-form, induced by integrating out all massive fields. After appropriate overall normalisation each of the massive fields yields an integer contribution to the Chern-Simons couplings. This is consistent with the topological nature of the Chern-Simons couplings that implies that their prefactors are quantised and turn out to be independent of the mass scale of the fields that are integrated out. As a consequence, they survive the limit in which the mass scale is taken to infinity. The results for the gauge Chern-Simons coupling were given in [5] without proof. This work substantiates our claims and extends them to include gravitational couplings. Our findings are summarised in section 2.

This analysis is not purely academic, since, remarkably, these couplings elegantly encode information about higher-dimensional anomalies after Kaluza-Klein compactification. In particular, we can consider a six-dimensional theory compactified on a circle to get a five-dimensional quantum field theory which propagates both massless and massive degrees of freedom. Six-dimensional gravitational anomalies are thus associated to the 
five-dimensional Chern-Simons couplings induced by integrating out excited Kaluza-Klein modes. Gauge anomalies can be accessed in the five-dimensional setup as well. In this case the massive degrees of freedom can also arise from spontaneous gauge symmetry breaking.

As already explained in [5] (see also [6]), a heuristic explanation for the above mentioned connection between five-dimensional Chern-Simons terms and six-dimensional anomalies can be obtained by considering the one-loop diagram necessary to determine the latter. Indeed, in six dimensions, the gravitational anomaly is captured by a four-point amplitude with external graviton legs. The polarisations of the latter have to be contracted in all possible ways and thus particularly also include contributions corresponding to the compact $S^{1}$ direction. From the five-dimensional perspective, the four-point function therefore decomposes into a sum of correlators involving (five-dimensional) gravitons, the Kaluza-Klein vector $A$, and the graviscalar, which we will consider to be non-dynamical and replace by the radius $r$. Focusing on terms which break five-dimensional parity, we are naturally lead to consider Chern-Simons terms of the form $A \wedge F \wedge F$ and $A \wedge \operatorname{tr}(R \wedge R)$, where $R$ is the five-dimensional curvature two-form and $F$ the field-strength tensor corresponding to $A$. One-loop corrections to these couplings are therefore expected to encode information about higher-dimensional anomalies. We will have more to say about this interesting connection in the upcoming paper [7].

There are various ways to embed our five-dimensional or six-dimensional setup into string theory and M-theory. We will consider two realisations in this work. Firstly, we will realise the five-dimensional setup by compactification of M-theory on an elliptically fibered Calabi-Yau threefold. Using the M-theory to F-theory limit reviewed in [8] the resulting low-energy effective action for the massless fields should be identified with the low energy effective action of a six-dimensional F-theory compactification on a circle after all KaluzaKlein modes are integrated out. The Chern-Simons couplings on the M-theory side are determined by the intersection numbers and the second Chern class of the Calabi-Yau threefold [9-11]. We thus find a purely geometric computation of the total Chern-Simons contribution for $(1,0)$ theories on a circle and agreement with our field theory computation can be shown.

A second string theory realisation of our setup can be found by considering heterotic string theory on $K 3 \times S^{1}$ in the absence of NS5-branes (see e.g. [12] for earlier computations in this setting). In this case the underlying six-dimensional $(1,0)$ theory is a theory with one self-dual and one anti-self dual tensor. The contributions to the five-dimensional ChernSimons terms can in this case be computed as a three-point amplitude at one-loop. It turns out that the computation is largely insensitive to most of the details of the internal world-sheet CFT which allows us to calculate the amplitudes explicitly for generic points of the moduli space of $K 3$ compactifications. The summation over the various massive modes propagating through the loop in the field theory picture corresponds to integrating over the moduli space of world-sheet tori from the string perspective that captures the contribution of all CFT excitations. We manage to perform this integration explicitly and find again agreement with our field theory result.

The paper is organised as follows. Section 2 summarises the main results of the paper. The families of massive fields that can generate one-loop Chern-Simons terms are listed 
in table 1, while table 2 gives the Chern-Simons coefficient for each of them. Section 3 contains the Feynman diagram computation of these coefficients. Section 4 discusses the check of the field theory results in the framework of six-dimensional F-theory compactifications. Section 5 is devoted to the explicit one-loop computation of the relevant amplitudes in heterotic string theory on $K 3 \times S^{1}$. Finally, in the conclusions we recapitulate our results and discuss briefly further directions. The main body of the paper is accompanied by several appendices. Notations, conventions, and other useful identities are collected in appendices A and B. The complete Feynman rules used in section 3 are gathered in appendix C. In appendix D we perform the calculation of a torus integral that appears in the string theory computation.

\section{Summary of results}

Let us start by summarising the results of this paper. The object of our investigation are five-dimensional theories in which some massive fields are coupled to a $\mathrm{U}(1)$ gauge field $A_{\mu}$ and to the metric $g_{\mu \nu}$. In particular, we study how quantum corrections due to massive fields can generate the Chern-Simons couplings

$$
S_{A F F}=k_{A F F} \int A \wedge F \wedge F, \quad S_{A R R}=k_{A R R} \int A \wedge \operatorname{tr}(R \wedge R)
$$

in the low energy effective action. In these expressions $F=d A$ is the field strength of the $\mathrm{U}(1)$ gauge field and $R$ denotes the curvature two-form built from the metric $g_{\mu \nu}$.

We show that three classes of massive fields are capable of generating such ChernSimons terms in the quantum effective action: massive spin- $1 / 2$ fermions $\psi$, massive selfdual tensors $B_{\mu \nu}$, and massive spin-3/2 fermions $\psi_{\mu}$. By massive self-dual tensor we mean a complex two-form $B_{\mu \nu}$ that admits a non-standard first order kinetic term $\bar{B} \wedge d B$ together with a mass term $m \bar{B} \wedge * B$. Its free equation of motion thus reads schematically

$$
* d B \propto m B .
$$

These tensor fields and their coupling to a $\mathrm{U}(1)$ gauge field has been analysed in $[5,13]$. Further details about massive self-dual tensors are given in section 3.1. We refer to these fields as self-dual because they can be thought of as the excited Kaluza-Klein modes of a six-dimensional self-dual tensor compactified on a circle.

Spin- $1 / 2$ fermions, self-dual tensors, and spin-3/2 fermions can be characterised in terms of associated representations of the massive little group in five dimensions, $\mathrm{SO}(4) \cong$ $\mathrm{SU}(2) \times \mathrm{SU}(2)$. Such representations are labelled by a pair of half-integer spins $\left(j_{1}, j_{2}\right)$. The correspondence between massive fields and $\mathrm{SO}(4)$ representations is summarised in table 1.

We have included the equation of motion that puts each field on-shell in the absence of interactions. The coefficients $c_{1 / 2}, c_{B}, c_{3 / 2}$ can take the values \pm 1 and determine which $\mathrm{SO}(4)$ representation is realised. Note that here and in the following $m$ denotes the mass of the physical one-particle states and is thus taken to be positive. The pairs of representations $\left(j_{1}, j_{2}\right)$ and $\left(j_{2}, j_{1}\right)$ are interchanged under parity. Correspondingly, these classes of fields 


\begin{tabular}{|ccc|}
\hline field & free EOM & $\mathrm{SO}(4)$ rep. \\
\hline spin-1/2 fermion $\psi$ & $\left(\not \partial-c_{1 / 2} m\right) \psi=0$ & $\left(\frac{1}{2}, 0\right)$ or $\left(0, \frac{1}{2}\right)$ \\
self-dual tensor $B_{\mu \nu}$ & $\left(* d-i c_{B} m\right) B=0$ & $(1,0)$ or $(0,1)$ \\
spin-3/2 fermion $\psi_{\mu}$ & $\left(\gamma^{\rho \mu \nu} \partial_{\mu}+c_{3 / 2} m \gamma^{\rho \nu}\right) \psi_{\nu}=0$ & $\left(\frac{1}{2}, 1\right)$ or $\left(1, \frac{1}{2}\right)$ \\
\hline
\end{tabular}

Table 1. Summary of massive representations considered in this work.

\begin{tabular}{|lccc|}
\hline & spin- $1 / 2$ fermion $\psi$ & self-dual tensor $B_{\mu \nu}$ & spin-3/2 fermion $\psi_{\mu}$ \\
\hline$k_{A F F}=$ & $-\frac{1}{48 \pi^{2}} q^{3} \cdot c_{1 / 2}$ & $-\frac{1}{48 \pi^{2}} q^{3} \cdot\left(-4 c_{B}\right)$ & $-\frac{1}{48 \pi^{2}} q^{3} \cdot\left(5 c_{3 / 2}\right)$ \\
$k_{A R R}=$ & $-\frac{1}{384 \pi^{2}} q \cdot c_{1 / 2}$ & $-\frac{1}{384 \pi^{2}} q \cdot\left(8 c_{B}\right)$ & $-\frac{1}{384 \pi^{2}} q \cdot\left(-19 c_{3 / 2}\right)$ \\
\hline
\end{tabular}

Table 2. Summary of the one-loop contributions for various fields.

break parity at tree level. From this point of view, the fact that couplings of the form (2.1) are generated in the effective action can be interpreted as a parity anomaly: quantum effects compensate for the parity violation originally induced by these families of massive fields, after they are integrated out.

Table 2 summarises our findings for the coefficients $k_{A F F}, k_{A R R}$ of the induced ChernSimons couplings in (2.1). Coefficients $c_{1 / 2}, c_{B}, c_{3 / 2}$ correspond to those in table 1 . The symbol $q$ denotes the $\mathrm{U}(1)$ charge of the massive fields. It is a dimensionless quantity and its normalisation is fixed by the minimal coupling prescription $\partial_{\mu} \rightarrow \partial_{\mu}-i q A_{\mu}$. The derivation of these results is the subject of the upcoming sections. Nonetheless, let us stress here two crucial aspects of the computation. Firstly, $k_{A F F}$ and $k_{A R R}$ are quantum corrected at one-loop only. This is expected by arguments involving locality of the effective action and quantisation of the Chern-Simons couplings [14] and is consistent with the interpretation in terms of parity anomalies in five dimensions.

Secondly, our results are derived using a simple quadratic action for the massive fields, which only includes minimal coupling to the gauge field $A_{\mu}$ and the metric $g_{\mu \nu}$. We argue that $k_{A F F}$ and $k_{A R R}$ are indeed insensitive to any fine detail of the interactions. For the $k_{A F F}$ coupling, the effect of some non-minimal interactions is analysed explicitly in section 3.4. It is shown there that such non-minimal couplings do not affect the renormalised value of $k_{A F F}$. These features are expected for topological couplings such as (2.1) that can be interpreted as parity anomalies.

Note that we refrain from a discussion about the possibility to write down fully consistent interacting theories for the three classes of massive fields under examination. For instance, it is expected that an interacting theory of massive spin- $3 / 2$ fermions is only possible in presence of (possibly spontaneously broken) supersymmetry, even though our 
findings are independent of the precise way it is realised in the five-dimensional action. From this point of view, we do not consider other parity-violating representations of $\mathrm{SO}(4)$, such as $\left(\frac{3}{2}, 0\right)$ or $(2,0)$, because no example is known of consistent interacting theories for the corresponding massive fields.

\section{$3 \quad$ Field theory computation}

In this section we compute the coefficients of the Chern-Simons couplings (2.1) in perturbative quantum field theory. We start by reviewing the actions for the massive spin- $1 / 2$ fermion, self-dual tensor, and spin- $3 / 2$ fermions minimally coupled to the $\mathrm{U}(1)$ gauge field and the metric. We then describe the main points of the Feynman diagram calculations for the gauge and the gravitational Chern-Simons terms. We conclude the section by studying the effect of some non-minimal couplings on the gauge Chern-Simons term.

\subsection{Minimally coupled massive actions}

The Chern-Simons couplings (2.1) can be captured by one-loop computations in a theory where the massive fields considered above are minimally coupled to the $\mathrm{U}(1)$ gauge field $A_{\mu}$ and the metric $g_{\mu \nu}$. In this section we briefly review the corresponding actions.

A spin- $1 / 2$ fermion is described by a five-dimensional Dirac spinor $\psi$. In order to couple it to the metric $g_{\mu \nu}$ we have to introduce a vielbein $e^{a}{ }_{\mu}$. The action for $\psi$ minimally coupled to the $\mathrm{U}(1)$ gauge field $A_{\mu}$ and the vielbein $e^{a}{ }_{\mu}$ is taken to be

$$
S_{1 / 2}=\int d^{5} x e\left[-\bar{\psi} \gamma^{\mu} \mathcal{D}_{\mu} \psi+c_{1 / 2} m \bar{\psi} \psi\right], \quad c_{1 / 2}= \pm 1,
$$

where $e=\operatorname{det} e^{a}{ }_{\mu}, \gamma^{\mu}=\gamma^{a} e_{a}{ }^{\mu}$, and where we have introduced the full spacetime and $\mathrm{U}(1)$ covariant derivative

$$
\mathcal{D}_{\mu} \psi=\partial_{\mu} \psi+\frac{1}{4} \omega_{\mu a b} \gamma^{a b} \psi-i q A_{\mu} \psi .
$$

On the right hand side, $\omega_{\mu a b}$ is the Levi-Civita spin connection constructed from the vielbein, and $q$ is the $\mathrm{U}(1)$ charge of the fermion $\psi$. More details about our spacetime and gamma-matrix conventions can be found in appendix A. As stated in section $2, m$ is the positive physical mass and $c_{1 / 2}$ labels two inequivalent spinor representations of the massive little group $\mathrm{SO}(4)$ in five dimensions. Under a parity transformation, the sign of $c_{1 / 2}$ is reversed.

Let us now turn to massive self-dual tensors in five-dimensions. Their action, including the coupling to a $\mathrm{U}(1)$ gauge field, can be written as [5]

$$
S_{B}=\int d^{5} x \sqrt{-g}\left[-\frac{1}{4} i c_{B} \epsilon^{\mu \nu \rho \sigma \tau} \bar{B}_{\mu \nu} \mathcal{D}_{\rho} B_{\sigma \tau}-\frac{1}{2} m \bar{B}_{\mu \nu} B^{\mu \nu}\right], \quad c_{B}= \pm 1
$$

The relevant part of the spacetime and $\mathrm{U}(1)$ covariant derivative reads

$$
\mathcal{D}_{[\rho} B_{\mu \nu]}=\partial_{[\rho} B_{\mu \nu]}-i q A_{[\rho} B_{\mu \nu]}
$$


Note that $g=\operatorname{det} g_{\mu \nu}$ and that $\epsilon^{\mu \nu \rho \sigma \tau}$ denotes the five-dimensional Levi-Civita tensor. In our conventions, it satisfies $\epsilon^{01234}=-1 / \sqrt{-g}$ if $0, \ldots, 4$ are curved indices. Note that in this case parity violation is not due to the mass term, but to the kinetic term.

Finally, a spin-3/2 fermion is described by a Dirac vector-spinor $\psi_{\mu}$ with action

$$
S_{3 / 2}=\int d^{5} x e\left[-\bar{\psi}_{\rho} \gamma^{\rho \mu \nu} \mathcal{D}_{\mu} \psi_{\nu}-c_{3 / 2} m \bar{\psi}_{\mu} \gamma^{\mu \nu} \psi_{\nu}\right], \quad c_{3 / 2}= \pm 1,
$$

where the antisymmetric part of the spacetime and $\mathrm{U}(1)$ covariant derivative is given by

$$
\mathcal{D}_{[\mu} \psi_{\nu]}=\partial_{[\mu} \psi_{\nu]}+\frac{1}{4} \omega_{[\mu \mid a b} \gamma^{a b} \psi_{\nu]}-i q A_{[\mu} \psi_{\nu]}
$$

In analogy with the spin- $1 / 2$ case, the two inequivalent representations of $\mathrm{SO}(4)$ differ by the sign of the mass term.

\subsection{Computation of the $A \wedge F \wedge F$ coupling}

The U(1) Chern-Simons coupling $A \wedge F \wedge F$ does not involve the gravitational field. As a consequence, throughout this section we can ignore the coupling of massive fields to gravity and take $g_{\mu \nu}=\eta_{\mu \nu}$. No distinction between flat and curved indices is made. The coupling to $A_{\mu}$ can be treated perturbatively in the framework of quantum field theory on flat spacetime.

The coefficient of the $A \wedge F \wedge F$ term in the quantum effective action can be extracted from the three-point function of the gauge field $A_{\mu}$. More precisely, we work in momentum space and we denote by $\Gamma_{A A A}$ the sum of 1PI Feynman diagrams with three external vectors with incoming momenta $p_{1}, p_{2}, p_{3}$ and polarisation vectors $e_{1}, e_{2}, e_{3}$. The Chern-Simons term

$$
k_{A F F} \int A \wedge F \wedge F=-k_{A F F} \int d^{5} x \epsilon^{\mu \nu \rho \sigma \tau} A_{\mu} \partial_{\nu} A_{\rho} \partial_{\sigma} A_{\sigma}
$$

in the effective action corresponds to a contribution to $\Gamma_{A A A}$ of the form

$$
\Gamma_{A A A} \supset i 3 ! \times\left(-k_{A F F}\right) \epsilon_{\lambda \tau \mu_{1} \mu_{2} \mu_{3}} p_{1}^{\lambda} p_{2}^{\tau} e_{1}^{\mu_{1}} e_{2}^{\mu_{2}} e_{3}^{\mu_{3}},
$$

where we have included a factor of $i$ from the Feynman rules and the combinatorial factor 3 ! to take into account symmetry under permutations of the three vectors. Contributions to $\Gamma_{A A A}$ different from (3.8) will be ignored. They correspond to higher-derivative and non-local terms in the effective action. As already mentioned, we expect that the right hand side of (3.8) is corrected at one loop only. As shown in sections 4 and 5 our one-loop results pass non-trivial tests in the framework of F-theory and heterotic string theory.

We can derive Feynman rules using the actions (3.1), (3.5) and (3.3) evaluated in flat spacetime and extract the propagators for massive fields, together with the interaction trivertex among two massive fields and one gauge field $A_{\mu}$. These propagators and vertices are listed in appendix $\mathrm{C}$.

At the one-loop level, only one class of diagrams can be built using the interaction vertices at hand. A representative diagram is depicted in figure 1. Wiggly lines represent the external vectors, while solid lines represent massive fields. Each class of massive fields 


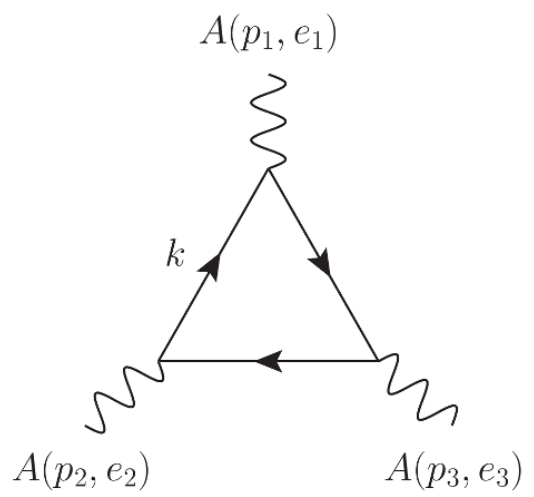

Figure 1. One-loop Feynman diagram involved in the computation of the Chern-Simons coefficient $k_{A F F}$. The external lines are three vectors $A$ with incoming momenta $p_{1}, p_{2}, p_{3}$ and polarisation vectors $e_{1}, e_{2}, e_{3}$. The internal lines can represent a massive spin- $1 / 2$ fermion, a massive self-dual tensor, or a massive spin- $3 / 2$ fermion. The loop momentum $k$ flows in the direction of the arrow.

contributes separately to the amplitude. To get the full answer, it has to be summed with the analog diagram where the orientation of the loop is reversed. This is equivalent to swapping the labels 1 and 2 on the external legs. Since the relevant structure in (3.8) is invariant under this relabelling, the loop-reversed diagram simply gives an overall additional factor 2 .

The denominator of the diagram (which is determined through its propagator factors) is the same for all fields running in the loop. If the labelling of figure 1 is adopted, it is given by

$$
\mathbb{D}=\frac{1}{k^{2}+m^{2}} \frac{1}{\left(k-p_{2}\right)^{2}+m^{2}} \frac{1}{\left(k+p_{1}\right)+m^{2}},
$$

which is to be completed by a suitable numerator factor $\mathbb{N}$ which particularly encodes information about the vertices and is strongly dependent on the fields running in the loop.

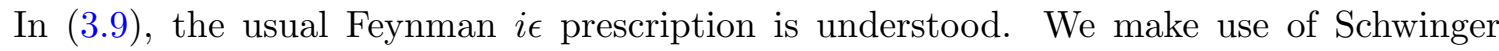
parameterisation to unify denominators, and write

$$
\mathbb{D}=\frac{1}{m^{6}} \int_{0}^{\infty} d \alpha \int_{0}^{\infty} d \beta \int_{0}^{\infty} d \gamma e^{-(\alpha+\beta+\gamma)\left(\ell^{2}+\Delta\right) / m^{2}} .
$$

In this expression, $\alpha, \beta, \gamma$ are dimensionless parameters, and we have made use of the shorthand notations

$$
\ell=k-y p_{2}+z p_{1}, \quad \Delta=m^{2}+2 y z p_{1} \cdot p_{2}+y(1-y) p_{1}^{2}+z(1-z) p_{2}^{2},
$$

where $y=\beta /(\alpha+\beta+\gamma)$ and $z=\gamma /(\alpha+\beta+\gamma)$. The full diagram is then given by

$$
\mathbb{I}=\mathbb{D} \cdot \mathbb{N}=\frac{1}{m^{6}} \int_{0}^{\infty} d \alpha \int_{0}^{\infty} d \beta \int_{0}^{\infty} d \gamma \int \frac{d^{5} \ell}{(2 \pi)^{5}} e^{-(\alpha+\beta+\gamma)\left(\ell^{2}+\Delta\right) / m^{2}} \mathbb{N},
$$

where, of course, the numerator is different for different species of massive fields running in the loop. We also note that the sum of the diagram in figure 1 with the diagram with the opposite orientation has a distinct symmetry with respect to exchanging the external 
points. On general grounds, one can show that this symmetries restrict the parity violating part of the integrand in (3.12) at the bilinear level in the external momenta to only depend on the Schwinger parameters in the combination $(\alpha+\beta+\gamma)$. This is a useful consistency check we have applied throughout the computations.

By naive power-counting arguments, we do not expect any infrared divergence in this one-loop diagram, but we cannot exclude the possibility of ultraviolet divergences. If Schwinger parameterisation is used, the integral over the loop momentum $\ell$ contains an exponential factor and (after Wick rotation) is convergent as long as $\alpha+\beta+\gamma$ is strictly positive. Ultraviolet divergences are translated into divergences in the $\alpha, \beta, \gamma$ integration, coming from the region where these three parameters are simultaneously small. We regularise the amplitude by cutting out this portion of the $\alpha, \beta, \gamma$ integration domain with a step-function: in (3.12) we make the replacement

$$
\int_{0}^{\infty} d \alpha \int_{0}^{\infty} d \beta \int_{0}^{\infty} d \gamma \rightarrow \int_{0}^{\infty} d \alpha \int_{0}^{\infty} d \beta \int_{0}^{\infty} d \gamma \theta(\alpha+\beta+\gamma-\epsilon)
$$

where $\epsilon>0$ is the regulator.

Recall from (3.8) that we are only interested in the coefficient of a term with two powers of external momenta contracted with an $\epsilon$-symbol. This allows us to simplify the computation of the diagram.

First of all, only the terms that contain an $\epsilon$-symbol have to be kept in the numerator. If a self-dual tensor runs in the loop, the $\epsilon$-symbol is introduced directly at the level of Feynman rules both in the propagator and in the vertex. When a spinor runs in the loop, the $\epsilon$-symbol is generated by traces of gamma matrices. This follows from the identities

$$
\operatorname{tr} 1=4, \quad \operatorname{tr} \gamma^{\mu_{1} \mu_{2} \mu_{3} \mu_{4} \mu_{5}}=4 i \epsilon^{\mu_{1} \mu_{2} \mu_{3} \mu_{4} \mu_{5}}, \quad \operatorname{tr} \gamma^{\mu_{1} \ldots \mu_{p}}=0 \text { for } p=1,2,3,4 .
$$

We see that only those terms need to be retained that contain an odd number of gamma matrices greater than or equal to five.

Second of all, we can perform a formal power series expansion of (3.12) in $p_{1}, p_{2}$ and we can neglect all terms that are not bilinear in $p_{1}$ and $p_{2}$. In particular, this implies that we can use the approximation $\Delta \approx m^{2}$, since all other terms in the exact expression (3.11) for $\Delta$ would generate additional powers of external momenta of the form $p_{1}^{2}, p_{2}^{2}$, or $p_{1} \cdot p_{2}$.

Finally, by symmetry arguments (not spoiled by our choice of regulator), we can make the following replacements in the numerator under the $\int d^{5} \ell$ integral:

$$
\begin{aligned}
& \ell_{\mu_{1}} \ldots \ell_{\mu_{r}} \rightarrow 0 \text { if } r \text { is odd } \\
& \ell_{\mu} \ell_{\nu} \rightarrow \frac{1}{5} \ell^{2} \eta_{\mu \nu}, \quad \ell_{\mu_{1}} \ell_{\mu_{2}} \ell_{\mu_{3}} \ell_{\mu_{4}} \rightarrow \frac{1}{35}\left(\ell^{2}\right)^{2}\left(\eta_{\mu_{1} \mu_{2}} \eta_{\mu_{3} \mu_{4}}+\eta_{\mu_{1} \mu_{3}} \eta_{\mu_{2} \mu_{4}}\right), \quad \ldots
\end{aligned}
$$

All tensor integrals in the loop momentum are thus reduced to scalar integrals.

The calculation of the diagram is now straightforward but tedious. ${ }^{1}$ After the numerator algebra is performed and the replacements (3.15) are made, the integrals over the loop

\footnotetext{
${ }^{1}$ We made use of the Mathematica packages xTensor of the bundle $x A c t$ [15] and GAMMA [16].
} 
momentum and the Schwinger parameters are computed using the formulae

$$
\begin{aligned}
& \int \frac{d^{5} \ell}{(2 \pi)^{5}} e^{-(\alpha+\beta+\gamma) \ell^{2} / m^{2}}\left(\ell^{2}\right)^{n}=\frac{i m^{2 n+5}}{24 \pi^{3}} \frac{\Gamma(n+5 / 2)}{(\alpha+\beta+\gamma)^{n+5 / 2}} \\
& \int_{0}^{\infty} d \alpha \int_{0}^{\infty} d \beta \int_{0}^{\infty} d \gamma \theta(\alpha+\beta+\gamma-\epsilon) \frac{e^{-(\alpha+\beta+\gamma)}}{(\alpha+\beta+\gamma)^{a}} \alpha^{n_{1}} \beta^{n_{2}} \gamma^{n_{3}}= \\
& =\frac{\Gamma\left(1+n_{1}\right) \Gamma\left(1+n_{2}\right) \Gamma\left(1+n_{3}\right)}{\Gamma\left(3+n_{1}+n_{2}+n_{3}\right)} \Gamma\left(3+n_{1}+n_{2}+n_{3}-a ; \epsilon\right) .
\end{aligned}
$$

We have performed the usual Wick rotation $\ell^{0} \rightarrow i \ell^{0}$ in the first integral and have introduced the incomplete gamma function

$$
\Gamma(x ; \epsilon)=\int_{\epsilon}^{\infty} d \tau \tau^{x-1} e^{-x}
$$

in the second integral.

Let us consider the diagram where the spin- $1 / 2$ fermion $\psi$ runs in the loop. By powercounting we expect a quadratic divergence, since the numerator has up to three powers of the loop momentum. The parity-violating part of the numerator, however, turns out to be of zero-th order in the loop momentum, thus giving a finite result without the need of any regulator.

This does not hold for the diagrams where $B_{\mu \nu}$ and $\psi_{\mu}$ run in the loop. In fact, even though the parity-violating part of the numerator has a better UV behaviour than the full diagram, it still contains terms proportional to $\ell^{2}$ or $\left(\ell^{2}\right)^{2}$. This implies that both diagrams have a divergent piece. In our regularisation scheme such divergences appear as coefficients of negative powers of the regulator $\epsilon$ in a formal expansion of the diagram.

We can then give the $\epsilon$-expansion for all the three species under consideration: spin- $1 / 2$ fermions $\psi$, tensors $B_{\mu \nu}$, and spin-3/2 fermions $\psi_{\mu}$,

$$
\begin{aligned}
& (\text { diagram })_{1 / 2}=\frac{i}{64 \pi^{2}} c_{1 / 2} q^{3}[ \\
& \left.+4+\mathcal{O}\left(\epsilon^{1 / 2}\right)\right] \\
& (\text { diagram })_{B}=\frac{i}{64 \pi^{2}} c_{B} q^{3}\left[\quad+\frac{15}{\sqrt{\pi}} \epsilon^{-1 / 2}-16+\mathcal{O}\left(\epsilon^{1 / 2}\right)\right], \\
& (\text { diagram })_{3 / 2}=\frac{i}{64 \pi^{2}} c_{1 / 2} q^{3}\left[-\frac{105}{4 \sqrt{\pi}} \epsilon^{-3 / 2}+\frac{15}{4 \sqrt{\pi}} \epsilon^{-1 / 2}+20+\mathcal{O}\left(\epsilon^{1 / 2}\right)\right] \text {. }
\end{aligned}
$$

Note that the factor $(-1)$ for a fermionic loop has been taken into account, but we have not inserted the overall factor 2 due to the diagram with the reversed loop orientation.

In order to extract the physical observable $k_{A F F}$ from these expressions we adopt a minimal subtraction prescription: negative powers of $\epsilon$ in the expansion are discarded. This gives the results of table 2. In section 3.4 we discuss the effect of non-minimal couplings and show how they can be used to cancel divergences.

\subsection{Computation of the $A \wedge \operatorname{tr}(R \wedge R)$ coupling}

Let us now turn to the discussion of the mixed U(1)-gravitational Chern-Simons term $A \wedge \operatorname{tr}(R \wedge R)$. To compute its coefficient we treat the coupling of massive fields to gravity 
perturbatively. The metric is written as

$$
g_{\mu \nu}=\eta_{\mu \nu}+h_{\mu \nu}
$$

and computations are performed order by order in a formal power series in $h_{\mu \nu}$ around flat spacetime. Indices $\mu, \nu, \ldots$ are thus raised and lowered with $\eta_{\mu \nu}$ and its inverse and no distinction is made between flat and curved indices. Further details about the expansion in $h_{\mu \nu}$ are collected in appendix B.

When $A \wedge \operatorname{tr}(R \wedge R)$ is expanded according to (3.22) terms with arbitrarily high powers of $h_{\mu \nu}$ are generated, because of the non-linear dependence of the Riemann tensor on the metric. Nonetheless, in order to read off the Chern-Simons coupling we can restrict to the lowest order term,

$$
\begin{aligned}
& k_{A R R} \int A \wedge \operatorname{tr}(R \wedge R)= \\
& =-\frac{1}{2} k_{A R R} \int d^{5} x \epsilon^{\mu_{1} \mu_{2} \mu_{3} \mu_{4} \mu_{5}} A_{\mu_{1}} \partial_{\lambda} \partial_{\mu_{2}} h_{\tau \mu_{3}}\left[\partial^{\tau} \partial_{\mu_{4}} h_{\mu_{5}}^{\lambda}-\partial^{\lambda} \partial_{\mu_{4}} h_{\mu_{5}}^{\tau}\right]+\mathcal{O}\left(h^{3}\right) .
\end{aligned}
$$

As a consequence, the constant $k_{A R R}$ can be extracted from the sum of 1PI Feynman diagrams with one vector and two gravitons, denoted $\Gamma_{A h h}$. More precisely, the sought-for Chern-Simons coupling corresponds to the contribution

$$
\Gamma_{A h h} \supset i 2 ! \times \frac{1}{2} k_{A R R} \epsilon_{\mu_{0} \mu_{1} \mu_{2} \lambda \tau} p_{1}^{\lambda} p_{2}^{\tau}\left(p_{1 \nu_{2}} p_{2 \nu_{1}}-\eta_{\nu_{1} \nu_{2}} p_{1} \cdot p_{2}\right) e_{0}^{\mu_{0}} e_{1}^{\mu_{1} \nu_{1}} e_{2}^{\mu_{2} \nu_{2}}
$$

where $p_{1}, p_{2}$ are the incoming momenta of the gravitons, $e_{0}$ is the polarisation tensor of the vector, and $e_{1}, e_{2}$ are the symmetric polarisation tensors of the gravitons. The prefactor $i 2$ ! comes from the standard Feynman rule prescriptions. Any term that does not match the structure of the right hand side of (3.24) will be neglected, since it would correspond to higher-derivative and non-local terms in the effective action.

It is interesting to note that the tensor structure in (3.24) is transverse with respect to both the vector and the graviton polarisation tensors, i.e. it vanishes if any of the replacements

$$
e_{0}^{\mu} \rightarrow p_{0}^{\mu}=-p_{1}^{\mu}-p_{2}^{\mu}, \quad e_{1}^{\mu \nu} \rightarrow a^{(\mu} p_{1}^{\nu)}, \quad e_{2}^{\mu \nu} \rightarrow a^{(\mu} p_{2}^{\nu)}
$$

is made, for arbitrary $a^{\mu}$. It can be shown that this tensor structure is the only structure with an $\epsilon$-symbol and four powers of external momenta that has this transversality property and is symmetric in the exchange of labels 1 and 2. Its appearance is a consequence of gauge invariance. Transversality with respect to $e_{0}$ reflects invariance of (3.23) under U(1) transformations. Transversality with respect to $e_{1}, e_{2}$ derives from invariance of (3.23) under diffeomorphisms. Recall that under an infinitesimal diffeomorphism with parameter $\xi^{\mu}$ we have

$$
\delta h_{\mu \nu}=2 \partial_{(\mu} \xi_{\nu)}+\mathcal{O}(h) .
$$

Gauge invariance can be used as a self-consistency check of the Feynman diagram computation. Indeed, we find that the desired contributions to $\Gamma_{A h h}$ organise into the structure (3.24) after all relevant diagrams are summed. 


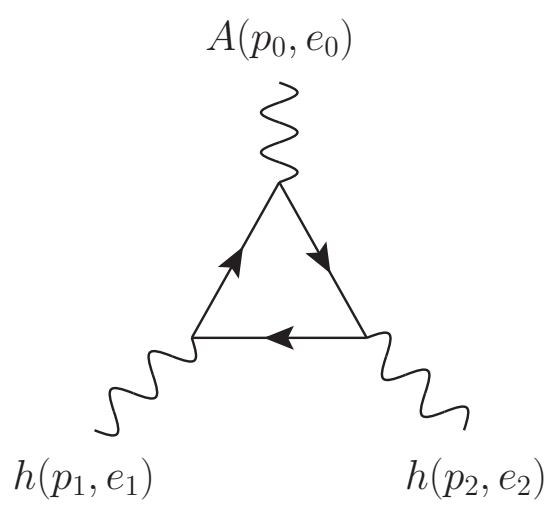

(a)

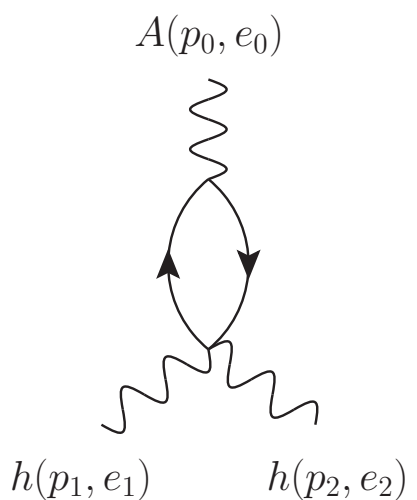

(b)

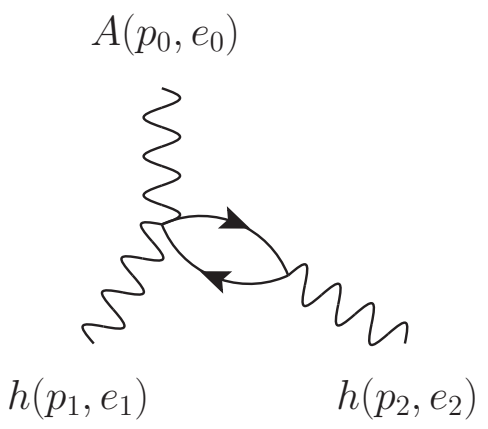

(c)

Figure 2. One-loop Feynman diagrams involved in the computation of the Chern-Simons coefficient $k_{A R R}$. The external line on top represents a vector $A$ with incoming momentum $p_{0}$ and polarisation vector $e_{0}$. The other external lines are gravitons $h$ with incoming momenta $p_{1}, p_{2}$ and symmetric polarisation tensors $e_{1}, e_{2}$. The internal lines can represent a massive spin- $1 / 2$ fermion, a massive self-dual tensor, or a massive spin- $3 / 2$ fermion.

The Feynman rules needed in the diagrammatic computation of $\Gamma_{A h h}$ are deduced by expanding the actions (3.1), (3.5), (3.3) for the massive fields according to (3.22). This gives interaction vertices of arbitrarily high powers in $h_{\mu \nu}$ but we only need an expansion up to second order in $h_{\mu \nu}$. More precisely, four kinds of vertices are relevant for the calculation of $\Gamma_{A h h}$. If we denote any of the massive fields $\psi, B_{\mu \nu}, \psi_{\mu}$ as $\Phi$, we need: the gauge trivertex $\bar{\Phi} \Phi A$, already considered in the previous section; the gravitational tri-vertex $\bar{\Phi} \Phi h$; the purely gravitational quadri-vertex $\bar{\Phi} \Phi h h$; the mixed gauge-gravitational quadri-vertex $\bar{\Phi} \Phi A h$. All such vertices are collected in appendix C.

The presence of quadri-vertices enlarges the family of one-loop Feynman diagrams that can be built. In particular, we have three different topologies, depicted in figure 2 . The total amplitude is given by the sum

$$
2(a)+(b)+2(c)
$$

where diagram (a) is counted twice because of the two possible orientations of the loop, 
and diagram (c) is counted twice according to which graviton is connected to the mixed quadri-vertex.

For each diagram, denominators can be unified by means of Schwinger parameters. In diagram (a) three parameters are needed, as in the previous section, while diagrams (b) and (c) require only two parameters. Up to minor changes, the methods described in the previous section can be applied straightforwardly to the diagrams at hand. In particular, UV divergences in diagrams (b) and (c) are regulated by means of the replacement

$$
\int_{0}^{\infty} d \alpha \int_{0}^{\infty} d \beta \rightarrow \int_{0}^{\infty} d \alpha \int_{0}^{\infty} d \beta \theta(\alpha+\beta-\epsilon)
$$

where $\alpha, \beta$ are the Schwinger parameters and $\epsilon$ is the regulator. For the sake of completeness, we record the two-parameter analog of the identity (3.17),

$$
\begin{aligned}
& \int_{0}^{\infty} d \alpha \int_{0}^{\infty} d \beta \theta(\alpha+\beta-\epsilon) \frac{e^{-(\alpha+\beta)}}{(\alpha+\beta)^{a}} \alpha^{n_{1}} \beta^{n_{2}}= \\
& =\frac{\Gamma\left(1+n_{1}\right) \Gamma\left(1+n_{2}\right)}{\Gamma\left(2+n_{1}+n_{2}\right)} \Gamma\left(2-a+n_{1}+n_{2} ; \epsilon\right) .
\end{aligned}
$$

Let us stress an important difference between the present computation and the one discussed in the previous section. In the case of the gauge Chern-Simons couplings, the relevant tensor structure (3.8) does not contain any product of external momenta. This allowed us to use the approximation $\Delta \approx m^{2}$ in the computation of the diagram in (3.12). In the present case, one of the two parts of the gauge invariant tensor structure (3.24) is proportional to $p_{1} \cdot p_{2}$. This implies that we have to keep the $p_{1} \cdot p_{2}$ term inside $\Delta$ and expand $e^{(\alpha+\beta+\gamma) \ell^{2} / m^{2}}$ (or $e^{(\alpha+\beta) \ell^{2} / m^{2}}$ ) in a power series in the external momenta. This is indeed crucial to obtain the gauge invariant structure (3.24) after all the three diagrams are combined according to (3.27).

As in the case of the gauge Chern-Simons term, the parity violating part of the diagrams has a better UV behaviour than expected from naive power-counting. Nevertheless, the diagrams in which the self-dual tensor and the spin-3/2 fermion run in the loop have some divergent parts. After all diagrams are summed according to (3.27) and the total expression is organised in powers of $\epsilon$, the $\epsilon^{0}$ coefficient is proportional to the gauge-invariant combination (3.24), while negative-power coefficients are not gauge-invariant. This leads us to apply a minimal subtraction prescription and simply drop the unphysical divergent pieces. In this way the results of table 2 are obtained.

Let us conclude this section with a side remark. Recall from section 3.2 that the relative weight between the diagram for spin- $1 / 2$ and spin- $3 / 2$ fermion contributions to $k_{A F F}$ is five. This result can be derived straightforwardly from an alternative form of the massive action for a spin-3/2 $\psi_{\mu}$,

$$
S_{3 / 2}^{\prime}=\int d^{5} x e\left[-\bar{\psi}_{\rho} \gamma^{\mu} \mathcal{D}_{\mu} \psi^{\rho}+c_{3 / 2} m \bar{\psi}_{\rho} \psi^{\rho}\right], \quad c_{3 / 2}= \pm 1
$$

Indeed, when this action is evaluated on a flat background, it gives exactly the same propagator and vertex as the spin- $1 / 2$ action (3.1), up to a factor of the metric $\eta_{\mu \nu}$. 
Remarkably, the alternative action (3.30) gives also the correct relative weight -19 between the spin- $1 / 2$ and the spin- $3 / 2$ contributions to $k_{A R R}$. This claim has been checked against an explicit Feynman diagram computation. To get the correct result is crucial to take into account the corrections to the vertices coming from the Christoffel symbols inside the covariant derivative $\mathcal{D}_{\mu} \psi^{\rho}$. Indeed, the vertices generated by the Christoffel symbol contribute a relative factor of -24 that combines with five times the spin- $1 / 2$ result to give -19 .

This finding resembles a similar result about gravitational anomalies in six dimensions [17]. In order to compute the contribution of a massless chiral spin-3/2 field $\psi_{\mu}$ to gravitational anomalies in six dimensions, one can use two different Lagrangians, proportional to

$$
\bar{\psi}_{\rho} \gamma^{\rho \mu \nu} \nabla_{\mu} \psi_{\nu} \quad \text { or } \quad \bar{\psi}_{\rho} \gamma^{\mu} \nabla_{\mu} \psi^{\rho}
$$

where $\nabla$ denotes the six-dimensional Levi-Civita covariant derivative. It is shown that the difference between these Lagrangians cannot affect the anomalous part of the four-graviton one-loop diagram. Note that if we compactify the six-dimensional Lagrangians (3.31) on a circle, the resulting actions for the massive Kaluza-Klein modes have kinetic and mass terms as given in (3.5) and (3.30), respectively. We are thus led to conjecture that corrections to the five-dimensional Chern-Simons terms (2.1) are insensitive to the precise form of the differential operator in the kinetic term and the corresponding form of the mass term.

\subsection{Non-minimal couplings and renormalisation}

The aim of this section is to describe the effect of non-minimal couplings on the ChernSimons term $A \wedge F \wedge F$, extending some remarks of [5]. Gravity is decoupled and the metric is taken to be $\eta_{\mu \nu}$. As far as fermions are concerned, we consider Pauli couplings built by contracting a spinor bilinear with the $\mathrm{U}(1)$ field strength $F=d A$. In particular, we have analysed the couplings

$$
\mathcal{L}_{1 / 2}^{\mathrm{nm}}=\frac{1}{2} i \tilde{q}_{1 / 2} F_{\mu \nu} \bar{\psi} \gamma^{\mu \nu} \psi, \quad \mathcal{L}_{3 / 2}^{\mathrm{nm}}=\frac{1}{2} i \tilde{q}_{3 / 2} F_{\mu \nu} \bar{\psi}_{\rho} \gamma^{\mu \nu \rho \sigma} \psi_{\sigma}+\frac{1}{2} i \tilde{q}_{3 / 2}^{\prime} F_{\mu \nu} \bar{\psi}^{\mu} \psi^{\nu}
$$

For massive self-dual tensors we have studied instead

$$
\mathcal{L}_{B}^{\mathrm{nm}}=\tilde{q}_{B} \bar{B}_{\mu \nu} F^{\nu \rho} B_{\rho}^{\mu}+\tilde{q}_{B}^{\prime} \bar{B}_{\mu \nu} F^{\nu \rho} B_{\rho \sigma} F^{\sigma \mu}
$$

The computation of section 3.2 can be repeated including these additional vertices. The corresponding Feynman rules can be obtained straightforwardly with the standard prescriptions. Note, however, that the coupling $\tilde{q}_{B}^{\prime}$ induces a quadri-vertex and therefore diagrams with a topology as diagram (b) or (c) in figure 2 have to be included.

We refrain from a detailed account on the computation. Nonetheless, its outcome is remarkable: all non-minimal couplings $\tilde{q}_{1 / 2}$ to $\tilde{q}_{B}^{\prime}$ drop from the $\epsilon^{0}$ coefficient of the combination of all diagrams and enter only the coefficients of negative powers in $\epsilon$.

This implies that they can be used to cancel divergences in the spin-3/2 and tensor diagrams. Recall from (3.21) that the triangle diagram with a spin-3/2 fermion running in the loop has two non-vanishing negative powers of $\epsilon$ if only the minimal coupling $q$ is 
switched on. Our computations reveal that turning $\tilde{q}_{3 / 2}, \tilde{q}_{3 / 2}^{\prime}$ on does not introduce higher negative powers, i.e. higher divergences, and does not affect the coefficient of the $\epsilon^{0}$ power. We can thus tune $\tilde{q}_{3 / 2}$ and $\tilde{q}_{3 / 2}^{\prime}$ and cancel divergences without altering the finite part of the diagram.

The same strategy can be applied to tensors. The reader might wonder why we take into account two non-minimal couplings for tensors, if the corresponding diagram has only one divergent part, as can be seen from (3.20). This is necessary since it can be checked that inclusion of the coupling $\tilde{q}_{B}$ introduces higher divergences that require the introduction of $\tilde{q}_{B}^{\prime}$ to be cancelled.

Our findings suggest the interpretation of non-minimal couplings (3.32) and (3.33) as counterterms. Dimensional analysis reinforces this claim, since it shows that non-minimal couplings $\tilde{q}_{1 / 2}$ to $\tilde{q}_{B}^{\prime}$ have negative mass dimension. In the limit in which the masses of $\psi, B_{\mu \nu}$, and $\psi_{\mu}$ tend to infinity and these fields are integrated out, non-minimal couplings are suppressed. A similar counterterm analysis for the gravitational Chern-Simons term is a formidable task and is not addressed in this work. Nevertheless, it is plausible that a similar mechanism can be implemented to cancel all divergences without changing the results of table 2 .

\section{Consistency with the M-theory to F-theory limit}

In this section we compare the field theory results summarised in table 2 with the predictions of five-dimensional compactifications of M-theory and F-theory. This will enable us to use geometric methods to perform consistency checks of the one-loop corrections. Our focus will be on the gauge and gravitational Chern-Simons actions in five-dimensional low energy effective supergravity theories with eight or sixteen supercharges. These arise, on the one hand, from M-theory compactifications on supersymmetry preserving threefolds. On the other hand, they arise from F-theory compactifications to six-dimensions preserving $(1,0)$ or $(2,0)$ supersymmetry if the theory is further compactified on a circle. The six-dimensional origin of five-dimensional effective theories with Chern-Simons terms is addressed in [7].

\subsection{Field theory prediction}

Let us apply the results of the one-loop computation of the previous section to the framework of $(1,0)$ and Abelian $(2,0)$ six-dimensional theories compactified on a circle. The field content of their supersymmetry multiplets is summarised in table 3 and features chiral fermions and (anti)self-dual tensors. The requirement of anomaly cancellation imposes some constraints on the spectrum of these theories. In particular, the absence of gravitational anomalies requires $[17,18]$

$$
\begin{array}{ll}
(1,0): & H-V=273-29 T, \\
(2,0): & T=21,
\end{array}
$$

where $T, V, H$ are the numbers of tensor multiplets, vector multiplets, and hypermultiplets, respectively. 


\begin{tabular}{|c|c|c|c|}
\hline \multicolumn{2}{|c|}{$(1,0)$ theory } & \multicolumn{2}{|c|}{$(2,0)$ theory } \\
\hline gravity multiplet & $\left(g_{\mu \nu}, B_{\mu \nu}^{+}, 2 \psi_{\mu}^{+}\right)$ & gravity multiplet & $\left(g_{\mu \nu}, 5 B_{\mu \nu}^{+}, 4 \psi_{\mu}^{+}\right)$ \\
\hline tensor multiplet & $\left(B_{\mu \nu}^{-}, \phi, 2 \psi^{-}\right)$ & tensor multiplet & $\left(B_{\mu \nu}^{-}, 5 \phi, 4 \psi^{-}\right)$ \\
\hline vector multiplet & $\left(A_{\mu}, 2 \psi^{+}\right)$ & & \\
\hline hypermultiplet & $\left(4 \phi, 2 \psi^{-}\right)$ & & \\
\hline
\end{tabular}

Table 3. Schematic form of supersymmetric spectra of $(1,0)$ and $(2,0)$ theories. The symbols $g_{\mu \nu}$, $B_{\mu \nu}, \psi, \phi$ represent the metric, a tensor, a Majorana-Weyl spinor, a real scalar field respectively. The prefactor counts the number of fields of a given species within each multiplet. The superscript \pm denotes (anti)self-duality for the tensors $B$ or chirality for the fermions $\psi$.

Upon compactification on a circle, the massive Kaluza-Klein modes of chiral fields are precisely given by the three families of massive fields summarised in table 1. More precisely, the excited modes of a symplectic Majorana-Weyl spinor are Dirac spinors and the modes of a (anti)self-dual tensor are massive complex self-dual tensors. We adopt conventions such that a positive chirality in six-dimensions correspond to a positive coefficient $c_{1 / 2}, c_{B}$, or $c_{3 / 2}$ in the mass term for excited Kaluza-Klein modes.

The ansatz for the metric reads

$$
d s_{6}^{2}=d s^{2}+r^{2}\left(d y-A^{0}\right)^{2}
$$

where $r$ is the circle radius and $A^{0}$ is the Kaluza-Klein vector. This choice of the sign of $A^{0}$ in the metric ansatz implies that an excited mode with dependence $e^{i n y}$ on the internal coordinate couples minimally to $A^{0}$ with $\mathrm{U}(1)$ covariant derivative $\partial_{\mu}+i n A_{\mu}^{0}$. This has to be contrasted with the minimal coupling prescription $\partial_{\mu}-i q A_{\mu}$ used in the loop computation. If we identify $A^{0}$ and $A$, we infer that $q=-n$ for the $n$-th Kaluza-Klein mode of any six-dimensional field.

In order to compute $k_{A F F}$ and $k_{A R R}$ defined in (2.1) we just have to sum the contributions of table 2 according to the spectra listed in 3 . For a $(1,0)$ theory, we have

$$
\begin{aligned}
& k_{A F F}^{(1,0)}=-\frac{1}{48 \pi^{2}} \sum_{n=1}^{\infty}(-n)^{3}[2(V-H-T)+2 \cdot 5+(1-T)(-4)]=-\frac{9-T}{24(2 \pi)^{2}}, \\
& k_{A R R}^{(1,0)}=-\frac{1}{384 \pi^{2}} \sum_{n=1}^{\infty}(-n)[2(V-H-T)+2 \cdot(-19)+(1-T)(+8)]=\frac{12-T}{24(2 \pi)^{2}},
\end{aligned}
$$

where we made use of the anomaly cancellation condition (4.1) and we employed zetafunction regularisations $\sum n^{3} \rightarrow \zeta(-3)=1 / 120$ and $\sum n \rightarrow \zeta(-1)=-1 / 12$ for the 
divergent sum over Kaluza-Klein levels. In a similar fashion, for a $(2,0)$ theory we find

$$
\begin{aligned}
& k_{A F F}^{(2,0)}=-\frac{1}{48 \pi^{2}} \sum_{n=1}^{\infty}(-n)^{3}[4(-T)+4 \cdot 5+(5-T)(-4)]=0, \\
& k_{A R R}^{(2,0)}=-\frac{1}{384 \pi^{2}} \sum_{n=1}^{\infty}(-n)[4(-T)+4 \cdot(-19)+(5-T)(+8)]=\frac{T+3}{96(2 \pi)^{2}}=\frac{1}{4(2 \pi)^{2}},
\end{aligned}
$$

where we recalled $T=21$ from (4.2). In the next subsection we will test these field theory predictions against geometric computation in the framework of M-theory and F-theory compactifications.

\section{$4.2 \quad$ F-theory check}

To begin with let us recall some facts about the F-theory reduction. Considering Ftheory on an elliptically fibered Calabi-Yau threefold $Y_{3}$ with full holonomy yields a sixdimensional $(1,0)$ supergravity theory. Non-Abelian gauge groups can originate from singularities of the fibre that are also singularities of the threefold itself. The singularities of the elliptic fibre signal the presence of spacetime filling seven-branes. In contrast, if one considers F-theory on a two-torus times a K3 surface, i.e. has a trivial elliptic fibration, then no seven-branes are present. The six-dimensional effective theory is a $(2,0)$ theory with 21 Abelian tensor multiplets.

Since there is no fundamental formulation of F-theory in twelve dimensions, the effective action of the six-dimensional theories has to be derived via the duality to M-theory [8]. This program was carried out for six-dimensional F-theory vacua in $[11,19]$. On the one side of the duality, the low-energy action of M-theory is reduced on $Y_{3}$ or $K 3 \times T^{2}$ down to five dimensions. In order for the low-energy action to be a viable approximation, the geometry of the compactification space has to be smooth. In particular, possible singularities of $Y_{3}$ associated to non-Abelian gauge groups must be resolved. At the level of the effective action, this implies that on the M-theory side of the duality we can only probe the five-dimensional Coulomb branch of the non-Abelian gauge theory. On the other side of the duality, we can use the constraints coming from $(1,0)$ or $(2,0)$ supersymmetry to parameterise the sought-for F-theory action in terms of a few key data. We then compactify this general six-dimensional action on a circle and we compute the low-energy action for the zero-modes in the Coulomb branch. By comparing the five-dimensional theories on the M-theory and F-theory side of the duality we can infer the data that determine the six-dimensional effective action of F-theory on $Y_{3}$ or $K 3 \times T^{2}$.

It is crucial to integrate out all massive fields in determining the low-energy effective action on the F-theory side. This implies that the M-theory action should encode in particular the information about the one-loop Chern-Simons couplings $A^{0} \wedge F^{0} \wedge F^{0}$ and $A^{0} \wedge \operatorname{tr}(R \wedge R)$. To check this claim we recall some facts about the reduction of the topological terms of the eleven-dimensional action on a Calabi-Yau threefold $Y_{3}$ and on $K 3 \times T^{2}$.

The relevant couplings in the eleven-dimensional action are the usual two-derivative Chern-Simons term together with a topological higher-derivative correction [20, 21]. Sup- 
pressing wedge products for brevity, we have

$$
S_{\text {top }}^{(11)}=\int\left[-\frac{1}{6} \frac{1}{(2 \pi)^{2}} C_{3} G_{4} G_{4}-\frac{1}{192} \frac{1}{(2 \pi)^{4}} C_{3}\left(\operatorname{tr} R^{4}-\frac{1}{4}\left(\operatorname{tr} R^{2}\right)^{2}\right)\right],
$$

where $C_{3}$ is the M-theory three-form, with mass dimension $3, G_{4}=d C_{3}$, and $R$ is the curvature two-form. This form of the action is consistent with the fact that $\int G_{4} /(2 \pi)$ is half-integrally quantised and that $\exp i S$ gives a well-defined functional in the path integral, once all terms of the effective action and the gravitino functional measure are taken into account [22]. This is crucial to match one-loop computations in field-theory, since the the standard Feynman rules are derived by an expansion of exp $i S_{\text {int }}$, fixing the absolute normalisation of one-loop induced Chern-Simons terms.

Let $X_{6}$ denote the internal space, for us $Y_{3}$ or $K 3 \times T^{2}$. The M-theory three-form is expanded on a basis $\left\{\omega_{A}\right\}$ of harmonic two-forms on $X_{6}$ as

$$
C_{3} \supset A^{A} \wedge \omega_{A}
$$

where $A^{A}$ are five-dimensional vectors. They have mass dimension one and their field strengths $F^{A}=d A^{A}$ are such that $\int F^{A} /(2 \pi)$ is integrally quantised. Dimensional reduction of the action (4.6) yields the five-dimensional topological terms [9-11]

$$
S^{\mathrm{CS}}=\frac{1}{(2 \pi)^{2}} \int\left[-\frac{1}{6} \mathcal{K}_{A B C} A^{A} F^{B} F^{C}+\frac{1}{96} c_{A} A^{A} \operatorname{tr} R^{2}\right],
$$

where we have introduced

$$
\mathcal{K}_{A B C}=\int_{X_{6}} \omega_{A} \wedge \omega_{B} \wedge \omega_{C}, \quad c_{A}=\int_{X_{6}} \omega_{A} \wedge c_{2}\left(X_{6}\right) .
$$

If $X_{6}=Y_{3}$, the Kaluza-Klein vector on the F-theory side is matched with the linear combination of vectors $A^{A}$ along the direction of the two-form

$$
\omega_{0}=\mathrm{PD}\left(B_{2}\right)+\frac{1}{2} c_{1}\left(B_{2}\right),
$$

where $\mathrm{PD}\left(B_{2}\right)$ is the Poincaré dual two form to the base $B_{2}$ of the elliptic fibration, and $c_{1}(B)$ is its first Chern class. ${ }^{2}$ The geometry of elliptically fibered Calabi-Yau threefolds ensures

$$
\begin{aligned}
\mathcal{K}_{000} & =\frac{1}{4} \int_{B_{2}} c_{1}\left(B_{2}\right)^{2}=\frac{1}{4}\left(10-h^{1,1}\left(B_{2}\right)\right), \\
c_{0} & =\int_{B_{2}}\left[c_{2}\left(B_{2}\right)+5 c_{1}\left(B_{2}\right)^{2}\right]=4\left(13-h^{1,1}\left(B_{2}\right)\right) .
\end{aligned}
$$

This in turn implies that the Chern-Simons sector of M-theory on $Y_{3}$ contains the terms

$$
S^{\mathrm{CS}} \supset \frac{1}{(2 \pi)^{2}} \int\left[-\frac{10-h^{1,1}\left(B_{2}\right)}{24} A^{0} F^{0} F^{0}+\frac{13-h^{1,1}\left(B_{2}\right)}{24} A^{0} \operatorname{tr} R^{2}\right] .
$$

\footnotetext{
${ }^{2}$ Strictly speaking one has to pull back $c_{1}(B)$ to $Y_{3}$, but we will suppress the pullback in the following.
} 
We just have to recall that the number of tensor multiplets of the $(1,0)$ theory is related to the geometry of $Y_{3}$ by

$$
h^{1,1}\left(B_{2}\right)=T+1
$$

to recognise a perfect match with the field theory prediction of the previous subsection.

In the case of compactification of M-theory on $X_{6}=K 3 \times T^{2}$, the Kaluza-Klein vector is identified with the vector along the only two-form on the torus, which we denote $\omega_{0}$. As a result,

$$
\mathcal{K}_{000}=0, \quad c_{0}=\int_{K 3 \times T^{2}} \omega_{0} \times c_{2}\left(K 3 \times T^{2}\right)=\int_{K 3} c_{2}(K 3)=24 .
$$

This implies that the gauge Chern-Simons term is absent, while the gravitational ChernSimons is given by

$$
S^{\mathrm{CS}} \supset \frac{1}{(2 \pi)^{2}} \int \frac{1}{4} A^{0} \operatorname{tr} R^{2}
$$

in agreement with the field theory computation.

So far we have focused on Chern-Simons coupling involving only the Kaluza-Klein vectors. There are additional terms in the reduction of M-theory on $Y_{3}$ that are interpreted as one-loop effects on the F-theory side. They are of the form

$$
k_{0 i j} \int A^{0} F^{i} F^{j}+k_{i j k} \int A^{i} F^{j} F^{k},
$$

where $A^{i}$ are the five-dimensional vectors that are lifted to six-dimensional vectors. The index $i$ labels the Cartan generators of the gauge group, since the duality between Mtheory and F-theory only works in the Coulomb phase. The coefficients $k_{0 i j}, k_{i j k}$ can be computed geometrically and are related to the charged spectrum of the theory, see for instance $[23,24]$.

To compute the coefficient of these couplings in field theory we need to consider diagrams where all massive fields charged under $A^{0}$ and/or $A^{i}$ run. Those are the Kaluza-Klein zeromodes and excited modes of the fields that acquire a mass after the gauge group is broken by giving a non-vanishing VEV to the scalars in the five-dimensional vector multiplets. We do not perform here a similar analysis, but we are confident about the applicability of the techniques developed so far to attack this problem. Note that it has indeed been shown in [23] that the Chern-Simons coefficient $k_{i j k}$ receives one-loop corrections by massive gauge degrees of freedom.

Let us close this section with a comment about a special case that recently attracted interest [25]. Namely, let us consider an M-theory compactification with $\chi\left(Y_{3}\right)=0$. When $Y_{3}$ is elliptically fibered one can lift the theory to a six-dimensional $(1,0)$ model. For simplicity, we assume that $Y_{3}$ has no gauge group singularities and hence the $(1,0)$ theory has no vector multiplets, $V=0$. In this case the Euler number is simply given by $\chi=$ $-60 \int_{B_{2}} c_{1}(B)^{2}=-60(9-T)$ and we see that $\chi=0$ implies $T=9$. The anomaly cancellation condition (4.1) requires then $H=12$. Can this model be interpreted as a spontaneously broken $(2,0)$ theory? Suppose we are given a possibly non-Abelian $(2,0)$ theory with 21 tensor multiplets, in accord with absence of gravitational anomalies. They correspond to 21 tensor multiplets and 21 hypermultiplets in $(1,0)$ language, as can be 
seen from table 3. Let us further imagine that the original theory undergoes a spontaneous supersymmetry breaking in such a way that only $T$ tensor multiplets out of 21 and and only $H$ hypermultiplets out of 21 remain massless. In order for the resulting $(1,0)$ theory to be free of gravitational anomalies, we must have $H=273-29 T$. The requirement $0 \leq H \leq 21$ together with the integrality of $T$ determines $T=9, H=12$ as the only possible breaking pattern. This agrees with the geometric setup with $\chi=0$. Furthermore, for $T=9$ we have $k_{A F F}^{(1,0)}=0$, see (4.4), and the term $A^{0} \wedge F^{0} \wedge F^{0}$, which is incompatible with 16 supersymmetries, does not enter the circle reduction of the $(1,0)$ theory. These might be considered as hints in favour of the spontaneous symmetry breaking scenario. If such breaking is actually possible, and how it may be realised, remains to be investigated.

\section{$5 \quad$ Dual heterotic string on $K 3 \times S^{1}$}

In this section we compare the gauge theory loop corrections derived in section 3 with a string loop computation performed in the heterotic string theory on $K 3 \times S^{1}$. The outcome of such computation only depends on topological data of $K 3$. A match with the F-theory setup of the previous section is expected on the basis of heterotic/F-theory duality for elliptically fibered $K 3$. We recall the basic five-dimensional setup in section 5.1 and present explicit one-loop corrections to the action in section 5.2.

\subsection{Heterotic setup}

The spectrum of heterotic string theory compactified on $K 3$ consists of a single tensor multiplet, $V$ vector multiplets and $H$ hypermultiplets coupled to the six-dimensional supergravity multiplet. The number of tensor multiplets is larger if we consider $K 3$ manifolds with singularities, however, we will not discuss this possibility.

Compactifying this theory further on $S^{1}$ (see [11]), the five-dimensional effective action contains $V^{(5)}=V+2$ physical vector multiplets and $H^{(5)}$ hypermultiplets coupled to the supergravity multiplet. The additional two vector multiplets come from the reduction of the single tensor multiplet and the six-dimensional supergravity multiplet. The scalar fields in these multiplets are the five-dimensional dilaton $\phi$ and the radius of $S^{1}$ which we will denote by $r$. The vector fields in turn correspond to the KK vectors coming from the reduction on $S^{1}$, i.e. from the reduction of the metric $\left(g_{\mu 6}\right)$ and the anti-symmetric B-field $\left(b_{\mu 6}\right)$ respectively. We follow [12] and denote these two as

$$
A_{\mu}=g_{\mu 6}+b_{\mu 6}, \quad B_{\mu}=g_{\mu 6}-b_{\mu 6},
$$

with their field strength denoted $F_{A, B}$ respectively. Finally, we denote the curvature 2 -form $R=d \omega+\omega \wedge \omega$, where $\omega$ is the spin-connection. $R$ is related to the Riemann tensor in the usual manner

$$
R_{b}^{a}=\frac{1}{2} \mathcal{R}_{b c d \mathfrak{e}^{c}}^{a} \mathfrak{e}^{d}, \quad \quad \text { with the vielbein } \quad \mathfrak{e}^{a}=e_{\mu}^{a} d x^{\mu},
$$

which will turn out to be more convenient for explicit calculations later on. 


\subsection{String amplitudes}

\subsubsection{Vertex operators}

Within the above setting we will particularly be interested to the one-loop corrections to the following terms in the string effective action

$$
\begin{aligned}
\mathcal{L}_{\mathrm{ST}}= & +\frac{1}{2} \mathcal{F}^{(A \mathcal{R} \mathcal{R})}(r) \epsilon^{\lambda \mu \nu \rho \tau} A_{\lambda} \mathcal{R}_{\beta \mu \nu}^{\alpha} \mathcal{R}^{\beta}{ }_{\alpha \rho \tau}+\frac{1}{2} \mathcal{F}^{(B \mathcal{R} \mathcal{R})}(r) \epsilon^{\lambda \mu \nu \rho \tau} B_{\lambda} \mathcal{R}_{\beta \mu \nu}^{\alpha} \mathcal{R}_{\alpha \rho \tau}^{\beta} \\
& +\frac{1}{6} \mathcal{F}^{\left(A F_{A} F_{A}\right)}(r) \epsilon^{\lambda \mu \nu \rho \tau} A_{\lambda} F_{A, \mu \nu} F_{A, \rho \tau}+\frac{1}{2} \mathcal{F}^{\left(B F_{A} F_{A}\right)}(r) \epsilon^{\lambda \mu \nu \rho \tau} B_{\lambda} F_{A, \mu \nu} F_{A, \rho \tau}
\end{aligned}
$$

where all coupling functions $\mathcal{F}$ are functions of the radius $r$. We will compute the corresponding one-loop scattering amplitudes using the RNS-formalism. In this approach the former are integrals over (higher-genus) Riemann surfaces and the external states are represented through emission vertex operators inserted at punctures, whose position needs to be integrated over the entire world-sheet. Thus, the first step is to discuss the precise form of the vertex operators for all states involved. We will use the same notation as in [12] and denote by $X^{\mu}(z)$ the embedding coordinates for the five spacetime directions and $X^{6}(z)$ the coordinate of the circle, while $\left(\psi^{\mu}(z), \psi^{6}(z)\right)$ are the corresponding (left-moving) superpartners. Here $z$ are two-dimensional coordinates on the world-sheet. With this notation, the graviton vertex operator in the 0 ghost picture is

$$
V_{\mathcal{R}}^{(0)}(h, p ; z)=h_{\mu \nu}\left[\partial X^{\mu}+i(p \cdot \psi) \psi^{\mu}\right] \bar{\partial} X^{\nu} e^{i p \cdot X},
$$

which is characterised by a symmetric, traceless polarisation tensor $h_{\mu \nu}$ and a fivemomentum $p_{\mu}$ such that $p^{\mu} h_{\mu \nu}=0$. The KK vector fields have vertex operators

$$
V_{A}^{(-1)}(\epsilon ; z)=\epsilon_{\mu} e^{-\varphi} \psi^{\mu} \bar{\partial} X^{6} e^{i p \cdot X}, \quad V_{B}^{(-1)}(\epsilon ; z)=\epsilon_{\mu} e^{-\varphi} \psi^{6} \bar{\partial} X^{\mu} e^{i p \cdot X}
$$

and are determined by the polarisation $\epsilon_{\mu}$. These vertices are written in the $(-1)$ picture, referring to the ghost system on the string world-sheet, which we have bosonised in terms of the scalar $\varphi$. In order to balance the ghost charge in a given amplitude, we also need picture changing operators $(\mathrm{PCO})$, the relevant part of which is given by

$$
V_{\mathrm{PCO}}=e^{\varphi} T_{F}, \quad T_{F}=\psi_{\mu} \partial X^{\mu}+\psi^{6} \partial X^{6}+T_{F}^{\mathrm{int}} .
$$

With these expressions we are in a position to compute explicit amplitudes.

\subsubsection{World-sheet CFT}

The effective couplings (5.3) can be related to explicit string amplitudes in the following manner

$$
\begin{aligned}
& \mathcal{F}^{(A \mathcal{R} \mathcal{R}, B \mathcal{R} \mathcal{R})}(r) \\
& =\int \frac{d^{2} \tau}{\tau_{2}^{2}} \int d^{2} z_{1,2,3}\left\langle V_{\mathcal{R}}^{(0)}\left(h^{(1)}, p^{(1)} ; z_{1}\right) V_{\mathcal{R}}^{(0)}\left(h^{(2)}, p^{(2)} ; z_{2}\right) V_{A, B}^{(-1)}\left(\epsilon, p ; z_{3}\right) V_{\mathrm{PCO}}\left(r_{0}\right)\right\rangle,
\end{aligned}
$$




$$
\begin{aligned}
& \mathcal{F}^{\left(A F_{A} F_{A}, B F_{A} F_{A}\right)}(r) \\
& =\int \frac{d^{2} \tau}{\tau_{2}^{2}} \int d^{2} z_{1,2,3}\left\langle V_{A}^{(0)}\left(\epsilon^{(1)}, p^{(1)} ; z_{1}\right) V_{A}^{(0)}\left(\epsilon^{(2)}, p^{(2)} ; z_{2}\right) V_{A, B}^{(-1)}\left(\epsilon^{(3)}, p^{(3)} ; z_{3}\right) V_{\mathrm{PCO}}\left(r_{0}\right)\right\rangle,
\end{aligned}
$$

where the integral over $\tau=\tau_{1}+i \tau_{2}$ runs over the fundamental domain of the world-sheet torus. Notice also that the position of the PCO $\left(r_{0}\right)$ is not integrated over, since the full correlator is independent of $r_{0}$. Since we work at one-loop, in principle, we have to take into account all different spin-structure configurations, corresponding to all possible boundary conditions of the world-sheet fermions along the two cycles of the torus. Fortunately, for these Chern-Simons like couplings only the odd spin-structure will be of relevance, since they involve contractions with $\epsilon^{\mu \nu \rho \sigma \tau}$. The correlators in (5.7) are understood within the full world-sheet theory. To calculate them, we first have to discuss the structure of the internal CFT where we essentially follow [26-28] (see also [29]). The partition function of $S^{1}$ is captured by the theta-series of the $\Gamma^{(1,1)}$ Narain lattice, whose momenta will be denoted $\left(P_{L}, P_{R}\right)$ and which are functions of the radius $r$ only. Concerning the remainder of the internal theory, the amplitudes (5.7) fortunately are not sensitive to the full details of the $K 3$ compactification, which would make it prohibitively difficult to compute them in general. In fact the only contribution is independent of the spin-structure and in the notation of [26-28] can be written in the form

$$
\bar{F}(\bar{\tau}):=d_{0} \frac{\bar{E}_{4}(\bar{\tau}) \bar{E}_{6}(\bar{\tau})}{\bar{\eta}^{24}}
$$

where we have also included the partition functions of the internal fermions and bosons. Here $\bar{E}_{2 k}$ for $k \geq 2$ are the anti-holomorphic Eisenstein series of weight $2 k$. The normalisation constant $d_{0}$ (which is not fixed through Köcher's principle alone) will be left undetermined in our computation and could be determined later by comparison to vector field amplitudes.

\subsubsection{Explicit amplitudes}

We now have all the ingredients to compute the amplitudes (5.7). By performing all contractions of the world-sheet fields, we find

$$
\begin{aligned}
& \mathcal{F}^{(A \mathcal{R} R)}=\frac{\pi^{3}}{3} \epsilon_{\lambda} p_{\alpha}^{(1)} p_{\beta}^{(2)} p_{\tau}^{(1)} p^{(2),[\nu} h_{\mu \nu}^{(1)} h_{\rho}^{(2), \tau]} \epsilon^{\alpha \mu \beta \rho \lambda} r \partial_{r} \mathcal{I}(r), \\
& \mathcal{F}^{(B \mathcal{R} \mathcal{R})}=\frac{\pi^{3}}{3} \epsilon_{\lambda} p_{\alpha}^{(1)} p_{\beta}^{(2)} p_{\tau}^{(1)} p^{(2),[\nu} h_{\mu \nu}^{(1)} h_{\rho}^{(2), \tau]} \epsilon^{\alpha \mu \beta \rho \lambda} \mathcal{I}(r), \\
& \mathcal{F}^{\left(A F_{A} F_{A}\right)}=64 \pi^{4} d_{0} \epsilon_{\mu}^{(1)} \epsilon_{\nu}^{(2)} \epsilon_{\rho}^{(3)} p_{\alpha}^{(1)} p_{\beta}^{(2)} \epsilon^{\mu \alpha \nu \beta \rho} r \partial_{r} \mathcal{I}_{0}(r), \\
& \mathcal{F}^{\left(B F_{A} F_{A}\right)}=64 \pi^{4} d_{0} \epsilon_{\mu}^{(1)} \epsilon_{\nu}^{(2)} \epsilon_{\rho}^{(3)} p_{\alpha}^{(1)} p_{\beta}^{(2)} \epsilon^{\mu \alpha \nu \beta \rho} \mathcal{I}_{0}(r),
\end{aligned}
$$


Here we have introduced the integral over the world-sheet torus

$$
\begin{aligned}
& \mathcal{I}_{0}(r):=\frac{i}{16 \pi^{2} d_{0}} \int \frac{d^{2} \tau}{\tau_{2}^{2}} F(\bar{\tau}) \partial_{\bar{\tau}}\left[\tau_{2}^{1 / 2} \sum_{\Gamma^{(1,1)}} q^{\frac{1}{2} P_{L}^{2}} \bar{q}^{\frac{1}{2} P_{R}^{2}}\right]=\frac{\theta(1-r) r^{3}}{3}+\frac{\theta(r-1)}{3 r^{3}} \\
& \mathcal{I}(r):=\int \frac{d^{2} \tau}{\tau_{2}^{3 / 2}} \hat{\bar{E}}_{2}(\bar{\tau}) \bar{F}(\bar{\tau}) \sum_{\Gamma^{(1,1)}} q^{\frac{1}{2} P_{L}^{2}} \bar{q}^{\frac{1}{2} P_{R}^{2}}= \\
&-8 d_{0} \pi\left[6\left(r+\frac{1}{r}\right)+5\left(r \theta(1-r)+\frac{\theta(r-1)}{r}\right)-2\left(r^{3} \theta(1-r)+\frac{\theta(r-1)}{r^{3}}\right)\right]
\end{aligned}
$$

where $\hat{E}_{2}(\tau, \bar{\tau})=E_{2}(\tau)-3 /\left(\pi \tau_{2}\right)$ is the quasi-holomorphic second Eisenstein series. $\mathcal{I}_{0}(r)$ was already evaluated in [12], while $\mathcal{I}(r)$ is performed in appendix D.

\subsection{Change of basis}

In order to make contact to [12] we will now make a change of basis of the vector fields. So far, in order to keep the computation of the loop amplitudes as simple as possible we have considered the vector fields in the basis (5.1). In order to physically interpret the couplings, however, we will now return to the basis $\left(g_{\mu 6}, b_{\mu 6}\right)$. The first couplings to be rewritten are the Chern-Simons terms

$$
\begin{aligned}
\mathcal{L}_{A F F}^{1-\text { loop }} & =\frac{1}{3 !} \mathcal{F}^{\left(A F_{A} F_{A}\right)}\left(A_{\mu} d x^{\mu}\right) \wedge F_{A} \wedge F_{A}+\frac{1}{2 !} \mathcal{F}^{\left(B F_{A} F_{A}\right)}\left(B_{\mu} d x^{\mu}\right) \wedge F_{A} \wedge F_{A} \\
& =\frac{2 \pi^{4} d_{0}}{3}\left[r \partial_{r} \mathcal{I}_{0}(r)\left(A_{\mu} d x^{\mu}\right)+3 \mathcal{I}_{0}(r)\left(B_{\mu} d x^{\mu}\right)\right] \wedge F_{A} \wedge F_{A}
\end{aligned}
$$

Upon changing to the basis

$$
A_{\mu}=A_{\mu}^{(1)} / r-A_{\mu}^{(2)} r, \quad B_{\mu}=A_{\mu}^{(1)} / r+A_{\mu}^{(2)} r
$$

the coupling (5.12) becomes ${ }^{3}$

$$
\begin{aligned}
\mathcal{L}_{A F F}^{1 \text {-loop }}= & 64 \pi^{4} d_{0}\left[a_{1}\left(A_{\mu}^{(1)} d x^{\mu}\right)+2\left(-a_{1} r^{2}+\frac{a_{2}}{2 r^{4}}\right)\left(A_{\mu}^{(2)} d x^{\mu}\right)\right] \wedge F^{(1)} \wedge F^{(1)}+ \\
& +64 \pi^{4} d_{0}\left[a_{2}\left(A_{\mu}^{(2)} d x^{\mu}\right)+2\left(-\frac{a_{2}}{r^{2}}+\frac{a_{1} r^{4}}{2}\right)\left(A_{\mu}^{(1)} d x^{\mu}\right)\right] \wedge F^{(2)} \wedge F^{(2)},
\end{aligned}
$$

where we have introduced (see [12])

$$
a_{1}=\theta(1-r) / 3, \quad a_{2}=\theta(r-1) / 3 .
$$

Similarly, we can also consider

$$
\begin{aligned}
\mathcal{L}_{F \mathcal{R}}^{\text {1-loop }} & =\frac{1}{2 !} \mathcal{F}^{(A \mathcal{R} \mathcal{R})}\left(A_{\mu} d x^{\mu}\right) \wedge \operatorname{Tr}(\mathcal{R} \wedge \mathcal{R})+\frac{1}{2 !} \mathcal{F}^{(B \mathcal{R} \mathcal{R})}\left(B_{\mu} d x^{\mu}\right) \wedge \operatorname{Tr}(\mathcal{R} \wedge \mathcal{R}) \\
& =-\frac{\pi^{3}}{3}\left[r \partial_{r} \mathcal{I}(r)\left(A_{\mu} d x^{\mu}\right)+\mathcal{I}(r)\left(B_{\mu} d x^{\mu}\right)\right] \wedge \operatorname{Tr}(\mathcal{R} \wedge \mathcal{R})
\end{aligned}
$$

\footnotetext{
${ }^{3}$ Notice that the explicit dependence on the compactification radius $r$ is $S^{1}$ is due to the fact that we are working in a frame in which the anti-symmetric two-form field has not been dualised into a vector.
} 
After the change of basis (5.13) this becomes

$$
\begin{aligned}
\mathcal{L}_{F \mathcal{R} \mathcal{R}}^{\text {1-loop }}= & -\frac{8 \pi^{4} d_{0}}{3}\left[6+15 a_{1}+6\left(-2 r^{2} a_{1}+\frac{a_{2}}{r^{4}}\right)\right]\left(A_{\mu}^{(1)} d x^{\mu}\right) \wedge \operatorname{Tr}(\mathcal{R} \wedge \mathcal{R}) \\
& -\frac{8 \pi^{4} d_{0}}{3}\left[6+15 a_{2}+6\left(-\frac{2 a_{2}}{r^{2}}+a_{1} r^{4}\right)\right]\left(A_{\mu}^{(2)} d x^{\mu}\right) \wedge \operatorname{Tr}(\mathcal{R} \wedge \mathcal{R}) .
\end{aligned}
$$

Extracting an overall factor of $-256 \pi^{6} d_{0}$, which is common to both couplings, ${ }^{4}$ we can read off

$$
k_{0}=-\frac{1}{12 \pi^{2}}, \quad \kappa_{0}=\frac{11}{96 \pi^{2}},
$$

in agreement with (4.4) for $T=1$, suitable for the heterotic compactification under study.

\section{Conclusions}

In this paper we have studied Chern-Simons terms in five-dimensional gauge theories with non-Abelian gauge groups. More precisely we have considered terms of the form $A^{0} \wedge F^{0} \wedge F^{0}$ and $A^{0} \wedge \operatorname{tr}(R \wedge R)$ where $A^{0}$ is a massless $\mathrm{U}(1)$ gauge field with field strength $F^{0}$ and $R$ is the curvature two-form. These terms are interesting for a number of reasons. Indeed, they appear as one-loop corrections to the low energy effective action by integrating out massive excited modes beyond a certain cutoff scale. While such contributions are usually suppressed in the limit of large cutoff and are therefore generically neglected, these ChernSimons terms are a rare example of a class of couplings that are independent of the scale introduced by the cutoff and thus have to be included. This property makes them very interesting for a number of applications: foremost, in a setting where the five-dimensional theory is obtained through compactification of a six-dimensional theory on a circle (and $A^{0}$ is identified with the Kaluza-Klein vector), we observe that these couplings encode information about the higher-dimensional gravitational anomalies. Thus, the Chern-Simons terms can be used as a tool to test whether a possible higher-dimensional parent theory is plagued by anomalies. We have more to say about the exciting possibility to use this connection to probe the quantum-consistency of a variety of five-dimensional gauge theories in an upcoming paper [7].

To compute these terms we have used three different approaches: first we have directly calculated them at the one-loop level in field theory. In this approach we have worked out the Feynman diagrams for massive spin- $1 / 2$, massive spin-3/2, and massive two-form tensors running in the loop. While these amplitudes are generically divergent, we have argued that it is possible to consistently introduce counterterms to get rid of all divergences. In the case of the gauge Chern-Simons term $A^{0} \wedge F^{0} \wedge F^{0}$, we have even made this procedure explicit. In a second step, we have successfully compared the field theoretic results with the predictions of five-dimensional M- and F-theory compactifications giving rise to theories with eight or sixteen supercharges. In this case, the couplings can be obtained from purely geometric considerations determining the spectrum of the five-dimensional effective theory. In a final step, using the duality between F-theory and heterotic string theory, we

\footnotetext{
${ }^{4}$ As already mentioned, we are not interested in the total overall normalisation of the effective action.
} 
have re-obtained the couplings through a one-loop computation in heterotic string theory compactified on $K 3 \times S^{1}$, where $K 3$ is realised as an elliptic fibration. Assuming that the latter has no singularities, the spectrum is limited to include only a single tensor multiplet. While we believe that it should in principle be possible to include more tensor multiplets, we have not further investigated this question in the current work. All three approaches in the end yield the same answer, which lends strong support to our understanding of Chern-Simons terms at the quantum level.

We expect further applications of our results in other fields of physics. For example, recent progress towards a description of the world-volume theory of multiple coincident M5-branes has been achieved by studying five-dimensional non-Abelian gauge theories including massive Kaluza-Klein modes [30-32]. We expect that Chern-Simons terms play an important role in determining the quantum consistency of these approaches and may also be relevant in extracting data of the M5-brane theory that are robust under dimensional reduction. For example, the conformal anomaly of $N$ coincident M5-branes shows a typical scaling behaviour of the form $N^{3}$ as was established using methods of the dual supergravity theory [33], or more recently from matrix model approaches [34-36] or other field theoretic methods [37, 38]. Non-perturbative topological string theory is yet another approach to study coincident M5-branes [39]. We expect that this scaling behaviour can also be extracted by studying certain classes of Chern-Simons terms involving a background metric (see [30] for some preliminary discussion).

Another possible application concerns higher-derivative corrections to black hole entropy in supergravity and string theory. Indeed, as discussed in [40-43], a gaugegravitational Chern-Simons term of the form $A \wedge \operatorname{tr}(R \wedge R$ ) (or rather its supersymmetric completion along the lines of [44]) plays a crucial role for the entropy and the attractor behaviour of five-dimensional black objects. Upon further compactification on a circle, these are also relevant four dimensional black holes. We thus expect our results to also be relevant for future studies of quantum corrections to black holes, in the supersymmetric as well as non-supersymmetric case.

Finally, we believe that results are not limited to five dimensions alone. Indeed, we expect similar relations between Chern-Simons terms in odd-dimensional theories to higherdimensional anomalies. For some recent examples in three dimensions see e.g. [6]. In the spirit of [7], Chern-Simons terms might be a useful tool in classifying a subclass of quantum consistent theories in any number of dimensions.

\section{Acknowledgments}

We would like to thank Luis Alvarez-Gaumé, Ignatios Antoniadis, Sophia Borowka, Thomas Hahn, Henrik Johansson, Denis Klevers, Neil Lambert, Noppadol Mekareeya, Boris Pioline, Tom Pugh, Raffaele Savelli, and Washington Taylor for useful discussions. The work of FB and TG was supported by a grant of the Max-Planck society. SH is grateful to the Ludwig-Maximilians-University and the Max-Planck Institute for Physics in Munich for kind hospitality during completion of this work. 


\section{A Notations and conventions}

We use Greek letters $\mu, \nu, \ldots$ for curved spacetime indices and Latin letters $a, b, \ldots$ for flat spacetime indices. They both take values $0, \ldots, 4$. (Anti)symmetrisation on any kind of indices is performed with weight one, e.g. $X_{(\mu \nu)}=\frac{1}{2} X_{\mu \nu}+\frac{1}{2} X_{\nu \mu}$. The metric $g_{\mu \nu}$, the vielbein $e^{a}{ }_{\mu}$, and the flat metric $\eta_{a b}$ are such that

$$
g_{\mu \nu}=\eta_{a b} e^{a}{ }_{\mu} e^{b}{ }_{\nu}, \quad \eta_{a b}=\operatorname{diag}(-,+,+,+,+) .
$$

We adopt the following conventions for the Christoffel symbol and the curvature tensors,

$$
\begin{aligned}
\Gamma_{\mu \nu}^{\rho} & =\frac{1}{2} g^{\rho \sigma}\left(\partial_{\mu} g_{\nu \sigma}+\partial_{\nu} g_{\mu \sigma}-\partial_{\sigma} g_{\mu \nu}\right), \\
R_{\tau \mu \nu}^{\lambda} & =\partial_{\mu} \Gamma_{\nu \tau}^{\lambda}-\partial_{\nu} \Gamma_{\mu \tau}^{\lambda}+\Gamma_{\mu \sigma}^{\lambda} \Gamma_{\nu \tau}^{\sigma}-\Gamma_{\nu \sigma}^{\lambda} \Gamma_{\mu \tau}^{\sigma} .
\end{aligned}
$$

The Levi-Civita tensor is denoted $\epsilon_{\mu \nu \rho \sigma \tau}$ and is such that

$$
\epsilon_{01234}=\sqrt{-g} \text { for curved indices } 0, \ldots, 4,
$$

where $g=\operatorname{det} g_{\mu \nu}$. The wedge product, Hodge star, and exterior derivative of differential forms satisfy

$$
\begin{aligned}
(\alpha \wedge \beta)_{\mu_{1} \ldots \mu_{p+q}} & =\frac{(p+q) !}{p ! q !} \alpha_{\left[\mu_{1} \ldots \mu_{p}\right.} \beta_{\left.\mu_{p+1} \ldots \mu_{p+q}\right]}, \quad(d \alpha)_{\mu_{0} \ldots \mu_{p}}=(p+1) \partial_{\left[\mu_{0}\right.} \alpha_{\left.\mu_{1} \ldots \mu_{p}\right]}, \\
(* \alpha)_{\mu_{1} \ldots \mu_{5-p}} & =\frac{1}{p !} \alpha^{\nu_{1} \ldots \nu_{p}} \epsilon_{\nu_{1} \ldots \nu_{p} \mu_{1} \ldots \mu_{5-p}},
\end{aligned}
$$

in which $\alpha$ is $p$-form and $\beta$ is a $q$-form.

The spin connection $\omega_{\mu a b}=\omega_{\mu[a b]}$ is determined by the vielbein $e^{a}{ }_{\mu}$ according to the torsionless condition

$$
d e^{a}+\omega^{a}{ }_{b} \wedge e^{b}=0 .
$$

The curvature two-form is given by

$$
R_{b}^{a}=d \omega_{b}^{a}+\omega_{c}^{a} \wedge \omega_{b}^{c}=\frac{1}{2} e_{\lambda}^{a} e_{b}{ }^{\tau} R_{\tau \mu \nu}^{\lambda} d x^{\mu} \wedge d x^{\nu},
$$

where in the last expression $R_{\tau \mu \nu}^{\lambda}$ denotes the Riemann tensor defined in (A.2).

Five-dimensional gamma matrices $\gamma^{a}$ are constant, complex-valued $4 \times 4$ matrices satisfying the anticommutation relation

$$
\left\{\gamma^{a}, \gamma^{b}\right\}=\eta^{a b}
$$

We always make use of the shorthand notation $\gamma^{a_{1} \ldots a_{p}}=\gamma^{\left[\mu_{1}\right.} \ldots \gamma^{\left.\mu_{p}\right]}$ and we work in a representation of gamma matrices such that

$$
\gamma^{a b c d e}=i \epsilon^{a b c d e} \mathbb{I}_{4} .
$$

The matrix $\gamma^{0}$ is taken to be anti-Hermitian, while $\gamma^{1}, \ldots \gamma^{4}$ are Hermitian. The Dirac conjugate of a spinor $\psi$ is defined as

$$
\bar{\psi}=\psi^{\dagger} \gamma^{0}
$$

Our spinors are Grassmann variables. Note that in our convention complex conjugation acts on products of Grassmann variables according to $(a b)^{*}=a^{*} b^{*}$. 


\section{B Gravitational perturbative expansion}

In this appendix we record some useful identities about the gravitational perturbative expansion around flat spacetime. More precisely, we assume a metric of the form

$$
g_{\mu \nu}=\eta_{\mu \nu}+h_{\mu \nu}
$$

and compute some geometrical quantities derived from the metric in a formal power series in $h_{\mu \nu}$. On the right hand side of the following identities, indices are raised and lowered with the flat metric $\eta_{\mu \nu}$ and its inverse. For instance, $h^{\mu \nu}=h_{\lambda \tau} \eta^{\lambda \mu} \eta^{\tau \nu}$.

The total inverse metric and volume form are given by

$$
\begin{aligned}
g^{\mu \nu} & =\eta^{\mu \nu}-h^{\mu \nu}+h^{\mu \lambda} h_{\lambda}{ }^{\nu}+\mathcal{O}\left(h^{3}\right) \\
\sqrt{-g} & =1+\frac{1}{2} h^{\mu}{ }_{\mu}+\frac{1}{8}\left(h^{\mu}{ }_{\mu} h^{\nu}{ }_{\nu}-2 h^{\mu \nu} h_{\mu \nu}\right)+\mathcal{O}\left(h^{3}\right) .
\end{aligned}
$$

The Christoffel symbols and the Riemann tensor are expanded as

$$
\begin{aligned}
\Gamma_{\mu \nu}^{\rho}= & \frac{1}{2}\left(\eta^{\rho \sigma}-h^{\rho \sigma}\right)\left(\partial_{\mu} h_{\nu \sigma}+\partial_{\nu} h_{\mu \sigma}-\partial_{\sigma} h_{\mu \nu}\right)+\mathcal{O}\left(h^{3}\right) \\
g_{\rho \tau} R_{\sigma \mu \nu}^{\tau}= & {\left[-\frac{1}{2} \partial_{\rho} \partial_{\mu} h_{\sigma \nu}-\frac{1}{8} \partial_{\lambda} h_{\mu \rho} \partial^{\lambda} h_{\nu \sigma}+\frac{1}{8} \partial_{\mu} h_{\sigma \lambda} \partial_{\nu} h_{\rho}{ }^{\lambda}-\frac{1}{4} \partial_{\rho} h_{\mu \lambda} \partial_{\nu} h_{\sigma}{ }^{\lambda}+\frac{1}{8} \partial_{\rho} h_{\nu \lambda} \partial_{\sigma} h_{\mu}{ }^{\lambda}\right.} \\
& \left.+\frac{1}{4} \partial_{\mu} h_{\rho \lambda} \partial^{\lambda} h_{\nu \sigma}-\frac{1}{4} \partial_{\rho} h_{\nu \lambda} \partial^{\lambda} h_{\mu \sigma}-(\mu \leftrightarrow \nu)\right]-(\rho \leftrightarrow \sigma)+\mathcal{O}\left(h^{3}\right) .
\end{aligned}
$$

In order to couple spinors to gravity we need to introduce a vielbein $e^{a}{ }_{\mu}$. It is determined by the metric only up to local Lorentz transformations. We fix this ambiguity by imposing the gauge condition

$$
\eta_{a b} \delta_{[\lambda}^{b} e_{\mu]}^{a}=0
$$

where the Kronecker delta plays the role of the vielbein for the flat metric $\eta_{\mu \nu}$. We thus find the following expansions of the vielbein and its inverse,

$$
\begin{aligned}
& e^{a}{ }_{\mu}=\eta^{a b} \delta_{b}{ }^{\lambda}\left[\eta_{\lambda \mu}+\frac{1}{2} h_{\lambda \mu}-\frac{1}{8} h_{\lambda \tau} h_{\mu}{ }^{\tau}+\mathcal{O}\left(h^{3}\right)\right] \\
& e_{a}{ }^{\mu}=\eta_{a b} \delta^{b}{ }_{\lambda}\left[\eta^{\lambda \mu}-\frac{1}{2} h^{\lambda \mu}+\frac{3}{8} h^{\lambda \tau} h^{\mu}{ }_{\tau}+\mathcal{O}\left(h^{3}\right)\right] .
\end{aligned}
$$

Finally, let us record the expansion of the flat components of the spin connection, which enter the fermion covariant derivatives:

$$
\begin{aligned}
e_{c}{ }^{\tau} \omega_{\tau a b}=\delta_{c}{ }^{\rho} \delta_{a}{ }^{\mu} \delta_{b}{ }^{\nu} & {\left[-\frac{1}{2} \partial_{\mu} h_{\nu \rho}-\frac{1}{2} h_{\nu \tau} \partial^{\tau} h_{\mu \rho}+\frac{1}{2} h_{\rho \tau} \partial_{\mu} h_{\nu}{ }^{\tau}\right.} \\
& \left.+\frac{1}{2} h_{\nu \tau} \partial_{\mu} h_{\rho}^{\tau}+\frac{1}{4} h_{\nu \tau} \partial_{\rho} h_{\mu}{ }^{\tau}-(\mu \leftrightarrow \nu)+\mathcal{O}\left(h^{3}\right)\right]
\end{aligned}
$$




\section{Feynman rules}

In this appendix we collect the Feynman rules that can be extracted from the massive actions (3.1), (3.5), (3.3). The propagators are read from the free actions where $A_{\mu}=0$ and $g_{\mu \nu}=\eta_{\mu \nu}$. The interaction vertices are obtained by expanding (3.1), (3.5), (3.3) up to second order in the metric perturbation $h_{\mu \nu}$, introduced in (3.22).

In all Feynman rules symmetrisation with weight one on graviton polarisation indices is understood. Moreover, the momenta of vectors and gravitons are always taken to be entering the vertex, while the momenta of massive fields flow in the same direction as specified by the charge arrow.

\section{C.1 Spin-1/2 fermion}
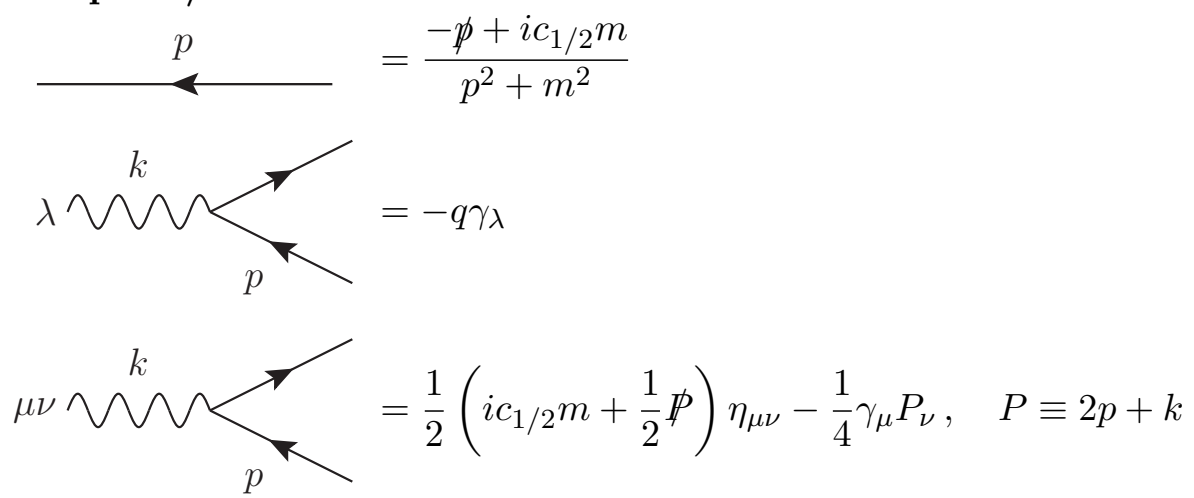

$$
\begin{aligned}
& \mu_{2} \nu_{2} \sim_{p_{2}}^{\mu_{1} \nu_{1}}=\frac{1}{4}\left(i c_{1 / 2} m+\frac{1}{2} \not p\right)\left(\eta_{\mu_{1} \nu_{1}} \eta_{\mu_{2} \nu_{2}}-2 \eta_{\mu_{1} \mu_{2}} \eta_{\nu_{1} \nu_{2}}\right) \\
& +\left[\frac{1}{16} \gamma_{\mu_{1} \mu_{2} \lambda} p_{1}^{\lambda}-\frac{1}{8} \gamma_{\mu_{1}} P_{\nu_{1}} \eta_{\mu_{2} \nu_{2}}+\frac{3}{16} \gamma_{\mu_{1}} P_{\mu_{2}} \eta_{\nu_{1} \nu_{2}}+(1 \leftrightarrow 2)\right], \\
& P \equiv 2 p+p_{1}+p_{2}
\end{aligned}
$$

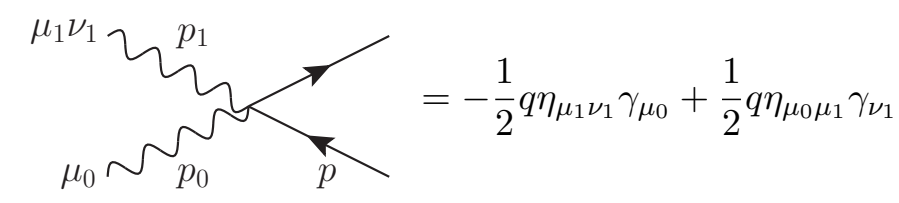

\section{C.2 Massive self-dual tensor}

In the following expression antisymmetrisation with weight one on tensor polarisation indices is understood.

$$
\rho \rho^{\prime} \overbrace{\sigma \sigma^{\prime}}^{p}=\frac{1}{p^{2}+m^{2}}\left\{-i c_{B} \epsilon_{\rho \rho^{\prime} \sigma \sigma^{\prime} \lambda} p^{\lambda}-2 i m \eta_{\rho \sigma} \eta_{\rho^{\prime} \sigma^{\prime}}-4 i m^{-1} \eta_{\rho \sigma} p_{\rho^{\prime}} p_{\sigma^{\prime}}\right\}
$$




$$
\begin{aligned}
& \rho \rho^{\prime} \\
& +\left[\frac{1}{2} i m \eta_{\mu_{1} \nu_{1}} \eta_{\mu_{2} \rho} \eta_{\nu_{2} \sigma} \eta_{\rho^{\prime} \sigma^{\prime}}-i m \eta_{\mu_{1} \mu_{2}} \eta_{\nu_{1} \rho} \eta_{\nu_{2} \sigma} \eta_{\rho^{\prime} \sigma^{\prime}}+(1 \leftrightarrow 2)\right] \\
& =-\frac{1}{8} i m\left(\eta_{\mu_{1} \nu_{1}} \eta_{\mu_{2} \nu_{2}}-2 \eta_{\mu_{1} \mu_{2}} \eta_{\nu_{1} \nu_{2}}\right) \eta_{\rho \sigma} \eta_{\rho^{\prime} \sigma^{\prime}}-i m \eta_{\mu_{1} \rho} \eta_{\nu_{1} \sigma} \eta_{\mu_{2} \rho^{\prime}} \eta_{\nu_{2} \sigma^{\prime}} \eta_{\rho^{\prime} \sigma^{\prime}}+i m \eta_{\mu \rho} \eta_{\nu \sigma} \eta_{\rho^{\prime} \sigma^{\prime}}
\end{aligned}
$$<smiles>CC(O)C(C=O)(C=O)C=O</smiles>

\section{C.3 Spin-3/2 fermion}

$$
\begin{aligned}
\rho \longleftarrow & p=\frac{1}{p^{2}+m^{2}}\left\{\left(\eta_{\rho \sigma}+\frac{p_{\rho} p_{\sigma}}{m^{2}}\right)\left(-\not p+i c_{3 / 2} m\right)\right. \\
& \left.+\frac{1}{4}\left(\gamma_{\rho}+\frac{i p_{\rho}}{c_{3 / 2} m}\right)\left(-\not p-i c_{3 / 2} m\right)\left(\gamma_{\sigma}+\frac{i p_{\sigma}}{c_{3 / 2} m}\right)\right\}
\end{aligned}
$$

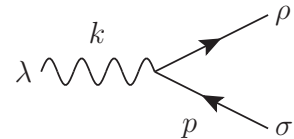

$=-q \gamma_{\rho \lambda \sigma}$

$\sim_{p}^{k}$

$$
=\frac{1}{2}\left(\frac{1}{2} \gamma_{\rho \lambda \sigma} P^{\lambda}-i c_{3 / 2} m \gamma_{\rho \sigma}\right) \eta_{\mu \nu}+\frac{1}{4} \gamma_{\rho \sigma \mu} P_{\nu}
$$

$-\frac{1}{4} \eta_{\mu \nu} \gamma_{\rho} k_{\sigma}+\frac{1}{4} \eta_{\mu \nu} \gamma_{\sigma} k_{\rho}+\frac{1}{4} \eta_{\mu \rho} \gamma_{\nu} k_{\sigma}-\frac{1}{4} \eta_{\mu \sigma} \gamma_{\nu} k_{\rho}-\frac{1}{4} \eta_{\mu \rho} \gamma_{\sigma} k_{\nu}+\frac{1}{4} \eta_{\mu \sigma} \gamma_{\rho} k_{\nu}$

$+\frac{1}{2}\left(\frac{1}{2} \gamma_{\sigma \lambda \mu} P^{\lambda}-i c_{3 / 2} m \gamma_{\sigma \mu}\right) \eta_{\nu \rho}-\frac{1}{2}\left(\frac{1}{2} \gamma_{\rho \lambda \mu} P^{\lambda}-i c_{3 / 2} m \gamma_{\rho \mu}\right) \eta_{\nu \sigma}$,

$$
P \equiv 2 p+k
$$

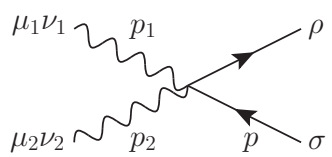

$=\frac{1}{4}\left(\frac{1}{2} \gamma_{\rho \lambda \sigma} P^{\lambda}-i c_{3 / 2} m \gamma_{\rho \sigma}\right)\left(\eta_{\mu_{1} \nu_{1}} \eta_{\mu_{2} \nu_{2}}-2 \eta_{\mu_{1} \mu_{2}} \eta_{\nu_{1} \nu_{2}}\right)$

$+\left[-\frac{1}{8} \gamma_{\rho}\left(p_{1}+p_{2}\right)_{\sigma}\left(\eta_{\mu_{1} \nu_{1}} \eta_{\mu_{2} \nu_{2}}-2 \eta_{\mu_{1} \mu_{2}} \eta_{\nu_{1} \nu_{2}}\right)\right.$

$+\frac{3}{8} i c_{3 / 2} m \gamma_{\sigma \mu_{1}} \eta_{\mu_{2} \rho} \eta_{\nu_{1} \nu_{2}}+\frac{1}{4} i c_{3 / 2} m \gamma_{\mu_{1} \mu_{2}} \eta_{\nu_{1} \sigma} \eta_{\nu_{2} \rho}$

$\left.-\frac{1}{8} \gamma_{\sigma \mu_{1} \mu_{2}} P_{\nu_{1}} \eta_{\nu_{2} \rho}+\frac{1}{8} \gamma_{\sigma \mu_{1} \mu_{2}} P_{\nu_{2}} \eta_{\nu_{1} \rho}+\frac{1}{8} \gamma_{\mu_{1} \mu_{2} \lambda} P^{\lambda} \eta_{\nu_{1} \rho} \eta_{\nu_{2} \sigma}-(\rho \leftrightarrow \sigma)\right]$

$+\left[-\frac{1}{16} \gamma_{\rho \sigma \mu_{1} \mu_{2} \lambda} p_{1}^{\lambda} \eta_{\nu_{1} \nu_{2}}+\frac{1}{8} \gamma_{\rho \sigma \mu_{1}} P_{\nu_{1}} \eta_{\mu_{2} \nu_{2}}-\frac{3}{16} \gamma_{\rho \sigma \mu_{1}} P_{\mu_{2}} \eta_{\nu_{1} \nu_{2}}+(1 \leftrightarrow 2)\right]$

$+\left[-\frac{1}{8} \gamma_{\sigma} p_{1 \mu_{1}} \eta_{\nu_{1} \rho} \eta_{\mu_{2} \nu_{2}}-\frac{1}{16} \not p_{1} \eta_{\mu_{1} \mu_{2}} \eta_{\nu_{1} \sigma} \eta_{\nu_{2} \rho}+\frac{3}{16} \gamma_{\sigma} p_{1 \mu_{2}} \eta_{\mu_{1} \rho} \eta_{\nu_{1} \nu_{2}}\right.$ 


$$
\begin{aligned}
& +\frac{5}{16} \gamma_{\sigma} p_{1 \mu_{1}} \eta_{\mu_{2} \rho} \eta_{\nu_{1} \nu_{2}}-\frac{1}{8} \gamma_{\mu_{2}} p_{1 \mu_{1}} \eta_{\nu_{1} \sigma} \eta_{\nu_{2} \rho}-\frac{1}{4} \gamma_{\sigma} p_{1 \mu_{2}} \eta_{\nu_{2} \rho} \eta_{\mu_{1} \nu_{1}} \\
& +\frac{1}{4} \gamma_{\mu_{1}} p_{1 \mu_{2}} \eta_{\nu_{2} \rho} \eta_{\nu_{1} \sigma}+\frac{1}{8} \gamma_{\mu_{2}} p_{1 \rho} \eta_{\mu_{1} \sigma} \eta_{\nu_{1} \nu_{2}}-\frac{1}{8} \gamma_{\mu_{1}} p_{1 \rho} \eta_{\nu_{1} \sigma} \eta_{\mu_{2} \nu_{2}} \\
& -\frac{1}{8} \gamma_{\mu_{2}} p_{1 \rho} \eta_{\mu_{2} \sigma} \eta_{\mu_{1} \nu_{1}}+\frac{1}{4} \gamma_{\mu_{1}} p_{1 \rho} \eta_{\mu_{2} \sigma} \eta_{\nu_{1} \nu_{2}}-\frac{1}{4} i c_{3 / 2} m \gamma_{\sigma \mu_{1}} \eta_{\nu_{1} \rho} \eta_{\mu_{2} \nu_{2}} \\
& \left.+\frac{3}{16} \gamma_{\sigma \mu_{2} \lambda} P^{\lambda} \eta_{\mu_{1} \rho} \eta_{\nu_{1} \nu_{2}}-\frac{1}{8} \gamma_{\sigma \mu_{1} \lambda} P^{\lambda} \eta_{\nu_{1} \rho} \eta_{\mu_{2} \nu_{2}}-(\rho \leftrightarrow \sigma)+(1 \leftrightarrow 2)\right], \\
P \equiv & 2 p+p_{1}+p_{2} \\
\underbrace{p_{p_{1}}}_{\mu_{1} \nu_{1}} \underbrace{}_{p_{0}} & -\frac{1}{2} q \eta_{\mu_{0} \mu_{1}} \gamma_{\nu_{1} \rho \sigma}+\frac{1}{2} q \eta_{\mu_{1} \nu_{1}} \gamma_{\mu_{0} \rho \sigma}-\frac{1}{2} q \eta_{\mu_{1} \rho} \gamma_{\mu_{0} \nu_{1} \sigma}+\frac{1}{2} q \eta_{\mu_{1} \sigma} \gamma_{\mu_{0} \nu_{1} \rho}
\end{aligned}
$$

\section{Torus integration}

In this appendix we perform the integral (5.11) over the world-sheet torus. It can be done in a straight-forward manner using lattice reduction techniques (see [45]). ${ }^{5}$ To this end, we begin by introducing the Siegel-Narain theta-function (without insertions) of the $\Gamma^{(1,1)}$ Narain lattice

$$
\Theta^{(1,1)}(\tau, \bar{\tau} ; P):=\sum_{\lambda \in \Gamma^{(1,1)}} e^{\pi i \tau\left(P_{+}(\lambda)\right)^{2}-\pi i \bar{\tau}\left(P_{-}(\lambda)\right)^{2}}
$$

and pick a basis of lattice vectors $\Gamma^{(1,1)}=\langle e, f\rangle_{\mathbb{Z}}$. Moreover, we view the Narain momenta introduced above as projections $P_{ \pm}$of the isometry $P: \Gamma \otimes \mathbb{R} \rightarrow \mathbb{R}^{1,1}$ to $\mathbb{R}^{1,0}$ and $\mathbb{R}^{0,1}$ respectively

$$
\begin{aligned}
& P_{+}(\lambda):=P_{L}=\frac{1}{\sqrt{2}}\left(\frac{m}{R}-n R\right), \\
& P_{-}(\lambda):=P_{R}=\frac{1}{\sqrt{2}}\left(\frac{m}{R}+n R\right), \quad \text { for } \quad \lambda=(n ; m) \in \Gamma^{(1,1)},
\end{aligned}
$$

where we have used the same parameterisation as in [12] in terms of the modulus $r$. Following the notation of [46], we can pick a primitive null vector $z \in \Gamma^{(1,1)}$ along which we can reduce $\Gamma^{(1,1)}$ to the trivial lattice. Upon denoting $z_{ \pm}:=P_{ \pm}(z)$ we get for (5.11) [45]

$$
\mathcal{I}(r)=\frac{\mathcal{I}_{\text {red }}}{\sqrt{2 z_{+}^{2}}}+\sqrt{\frac{2}{z_{+}^{2}}} \sum_{\ell=1}^{\infty} \sum_{t=0}^{1} c(0, t)\left(\frac{2 z_{+}^{2}}{\pi \ell^{2}}\right)^{t+1} \Gamma(t+1)
$$

Here $\mathcal{I}_{\text {red }}$ is another torus integral, however, without a Siegel-Narain theta function in the integrand

$$
\mathcal{I}_{\text {red }}=\frac{1}{2 \zeta(2)} \int \frac{d^{2} \tau}{\tau_{2}^{2}} \hat{\bar{G}}_{2}(\tau, \bar{\tau}) \bar{F}(\bar{\tau})=\left.\frac{3}{2 \pi^{3}}\left[\bar{G}_{2}^{2}(\bar{\tau}) \bar{F}(\bar{\tau})\right]\right|_{\bar{q}^{0}}=-48 \pi d_{0}
$$

with $G_{2 k}:=2 \zeta(2 k) E_{2 k}$. Moreover, the information about the modular form $\bar{F}(\bar{\tau})$ enters into (D.3) through the coefficients $c(0, t)$. To be precise, they are the Fourier coefficients

\footnotetext{
${ }^{5}$ Other techniques for computing such integrals can be found in [47-53].
} 
of the expansion of the following modular invariant function

$$
\hat{E}_{2}(\bar{\tau}) F(\bar{\tau})=\sum_{k=-1}^{\infty} \sum_{t=0}^{1} c(k, t) \bar{q}^{k} \tau_{2}^{-t} .
$$

Before giving the explicit expression for $\mathcal{I}(r)$, there is one more crucial point which needs mentioning. The expression (D.3) is only valid under the assumption that $z_{+}$is sufficiently small. Since $z_{+}$(through the projection $P_{+}$) depends on the modulus $r$, a particular choice of $z$ will only be valid in a certain region of the moduli space (chamber dependence). Therefore, in order to cover all chambers, we need to consider reductions along different vectors. A convenient choice is to set $z=e$, in which case (see [46]) $z_{+}^{2}=\frac{r^{2}}{2}$ which is a valid choice for $r<1$. From this we can deduce the result in the chamber $r>1$ by exchanging $r \leftrightarrow \frac{1}{r}$. The full answer then takes the form

$$
\begin{aligned}
\mathcal{I}(r) & =-d_{0} \pi \theta(1-r)\left[\frac{48}{r}-2 \sum_{t=0}^{1} \frac{\zeta(2+2 t) \Gamma(t+1)}{\pi^{t+2}} \frac{c(0, t)}{d_{0}} r^{2 t+1}\right]+\left(r \leftrightarrow \frac{1}{r}\right) \\
& =-8 d_{0} \pi\left[6\left(r+\frac{1}{r}\right)+5\left(r \theta(1-r)+\frac{\theta(r-1)}{r}\right)-2\left(r^{3} \theta(1-r)+\frac{\theta(r-1)}{r^{3}}\right)\right]
\end{aligned}
$$

This expression is also consistent with the expression for $\mathcal{I}_{0}$ in (5.10) first computed in [12].

Open Access. This article is distributed under the terms of the Creative Commons Attribution License which permits any use, distribution and reproduction in any medium, provided the original author(s) and source are credited.

\section{References}

[1] K. Symanzik, Infrared singularities and small distance behavior analysis, Commun. Math. Phys. 34 (1973) 7 [INSPIRE].

[2] T. Appelquist and J. Carazzone, Infrared Singularities and Massive Fields, Phys. Rev. D 11 (1975) 2856 [INSPIRE].

[3] J. Goldstone and F. Wilczek, Fractional Quantum Numbers on Solitons, Phys. Rev. Lett. 47 (1981) 986 [INSPIRE].

[4] E. D'Hoker and E. Farhi, Decoupling a Fermion Whose Mass Is Generated by a Yukawa Coupling: The General Case, Nucl. Phys. B 248 (1984) 59 [INSPIRE].

[5] F. Bonetti, T.W. Grimm and S. Hohenegger, A Kaluza-Klein inspired action for chiral p-forms and their anomalies, Phys. Lett. B 720 (2013) 424 [arXiv:1206.1600] [INSPIRE].

[6] M. Cvetič, T.W. Grimm and D. Klevers, Anomaly Cancellation And Abelian Gauge Symmetries In F-theory, JHEP 02 (2013) 101 [arXiv: 1210.6034] [INSPIRE].

[7] F. Bonetti, T.W. Grimm and S. Hohenegger, Exploring $6 D$ origins of 5D supergravities with Chern-Simons terms, JHEP 05 (2013) 124 [arXiv:1303.2661] [INSPIRE].

[8] F. Denef, Les Houches Lectures on Constructing String Vacua, arXiv:0803.1194 [INSPIRE]. 
[9] A. Cadavid, A. Ceresole, R. D'Auria and S. Ferrara, Eleven-dimensional supergravity compactified on Calabi-Yau threefolds, Phys. Lett. B 357 (1995) 76 [hep-th/9506144] [INSPIRE].

[10] I. Antoniadis, S. Ferrara, R. Minasian and K. Narain, $R^{4}$ couplings in $M$ and type-II theories on Calabi-Yau spaces, Nucl. Phys. B 507 (1997) 571 [hep-th/9707013] [INSPIRE].

[11] F. Bonetti and T.W. Grimm, Six-dimensional $(1,0)$ effective action of F-theory via M-theory on Calabi-Yau threefolds, JHEP 05 (2012) 019 [arXiv:1112.1082] [INSPIRE].

[12] I. Antoniadis, S. Ferrara and T. Taylor, N=2 heterotic superstring and its dual theory in five-dimensions, Nucl. Phys. B 460 (1996) 489 [hep-th/9511108] [INSPIRE].

[13] P. Townsend, K. Pilch and P. van Nieuwenhuizen, Selfduality in Odd Dimensions, Phys. Lett. B 136 (1984) 38 [Addendum ibid. B 137 (1984) 443] [INSPIRE].

[14] E. Witten, Phase transitions in M-theory and F-theory, Nucl. Phys. B 471 (1996) 195 [hep-th/9603150] [INSPIRE].

[15] José M. Martín-García, xAct: Efficient tensor computer algebra for Mathematica, GPL 2002-2013, http://www.xact.es/index.html.

[16] U. Gran, GAMMA: A Mathematica package for performing gamma matrix algebra and Fierz transformations in arbitrary dimensions, hep-th/0105086 [INSPIRE].

[17] L. Álvarez-Gaumé and E. Witten, Gravitational Anomalies, Nucl. Phys. B 234 (1984) 269 [INSPIRE].

[18] W. Taylor, TASI Lectures on Supergravity and String Vacua in Various Dimensions, arXiv:1104.2051 [INSPIRE].

[19] S. Ferrara, R. Minasian and A. Sagnotti, Low-energy analysis of $M$ and $F$ theories on Calabi-Yau threefolds, Nucl. Phys. B 474 (1996) 323 [hep-th/9604097] [INSPIRE].

[20] C. Vafa and E. Witten, A One loop test of string duality, Nucl. Phys. B 447 (1995) 261 [hep-th/9505053] [INSPIRE].

[21] M. Duff, J.T. Liu and R. Minasian, Eleven-dimensional origin of string-string duality: A One loop test, Nucl. Phys. B 452 (1995) 261 [hep-th/9506126] [INSPIRE].

[22] E. Witten, On flux quantization in M-theory and the effective action, J. Geom. Phys. 22 (1997) 1 [hep-th/9609122] [INSPIRE].

[23] K.A. Intriligator, D.R. Morrison and N. Seiberg, Five-dimensional supersymmetric gauge theories and degenerations of Calabi-Yau spaces, Nucl. Phys. B 497 (1997) 56 [hep-th/9702198] [INSPIRE].

[24] T.W. Grimm and H. Hayashi, F-theory fluxes, Chirality and Chern-Simons theories, JHEP 03 (2012) 027 [arXiv: 1111.1232] [INSPIRE].

[25] A.-K. Kashani-Poor, R. Minasian and H. Triendl, Enhanced supersymmetry from vanishing Euler number, JHEP 04 (2013) 058 [arXiv: 1301.5031] [INSPIRE].

[26] I. Antoniadis, E. Gava, K. Narain and T. Taylor, Topological amplitudes in string theory, Nucl. Phys. B 413 (1994) 162 [hep-th/9307158] [INSPIRE].

[27] I. Antoniadis, S. Hohenegger, K. Narain and E. Sokatchev, A New Class of $N=2$ Topological Amplitudes, Nucl. Phys. B 823 (2009) 448 [arXiv:0905.3629] [INSPIRE]. 
[28] I. Antoniadis, S. Hohenegger, K. Narain and E. Sokatchev, Generalized N $=2$ Topological Amplitudes and Holomorphic Anomaly Equation, Nucl. Phys. B 856 (2012) 360 [arXiv:1107.0303] [INSPIRE].

[29] S. Hohenegger and S. Stieberger, BPS Saturated String Amplitudes: K3 Elliptic Genus and Igusa Cusp Form, Nucl. Phys. B 856 (2012) 413 [arXiv:1108.0323] [InSPIRE].

[30] F. Bonetti, T.W. Grimm and S. Hohenegger, Non-Abelian Tensor Towers and (2,0) Superconformal Theories, JHEP 05 (2013) 129 [arXiv:1209.3017] [INSPIRE].

[31] P.-M. Ho, K.-W. Huang and Y. Matsuo, A Non-Abelian Self-Dual Gauge Theory in 5+1 Dimensions, JHEP 07 (2011) 021 [arXiv:1104.4040] [INSPIRE].

[32] K.-W. Huang, Non-Abelian Chiral 2-Form and M5-Branes, arXiv:1206.3983 [INSPIRE].

[33] F. Bastianelli, S. Frolov and A.A. Tseytlin, Conformal anomaly of (2,0) tensor multiplet in six-dimensions and AdS/CFT correspondence, JHEP 02 (2000) 013 [hep-th/0001041] [INSPIRE].

[34] J. Kallen, J. Qiu and M. Zabzine, The perturbative partition function of supersymmetric $5 D$ Yang-Mills theory with matter on the five-sphere, JHEP 08 (2012) 157 [arXiv:1206.6008] [INSPIRE].

[35] J. Kallen, J. Minahan, A. Nedelin and M. Zabzine, $N^{3}$-behavior from $5 D$ Yang-Mills theory, JHEP 10 (2012) 184 [arXiv:1207.3763] [INSPIRE].

[36] D.L. Jafferis and S.S. Pufu, Exact results for five-dimensional superconformal field theories with gravity duals, arXiv:1207.4359 [INSPIRE].

[37] P. Yi, Anomaly of (2,0) theories, Phys. Rev. D 64 (2001) 106006 [hep-th/0106165] [INSPIRE].

[38] T. Maxfield and S. Sethi, The Conformal Anomaly of M5-Branes, JHEP 06 (2012) 075 [arXiv: 1204.2002] [INSPIRE].

[39] G. Lockhart and C. Vafa, Superconformal Partition Functions and Non-perturbative Topological Strings, arXiv:1210.5909 [INSPIRE].

[40] A. Castro, J.L. Davis, P. Kraus and F. Larsen, 5D attractors with higher derivatives, JHEP 04 (2007) 091 [hep-th/0702072] [INSPIRE].

[41] A. Castro, J.L. Davis, P. Kraus and F. Larsen, 5D Black Holes and Strings with Higher Derivatives, JHEP 06 (2007) 007 [hep-th/0703087] [INSPIRE].

[42] A. Castro, J.L. Davis, P. Kraus and F. Larsen, Precision Entropy of Spinning Black Holes, JHEP 09 (2007) 003 [arXiv:0705.1847] [INSPIRE].

[43] A. Castro, J.L. Davis, P. Kraus and F. Larsen, String Theory Effects on Five-Dimensional Black Hole Physics, Int. J. Mod. Phys. A 23 (2008) 613 [arXiv:0801.1863] [InSPIRE].

[44] K. Hanaki, K. Ohashi and Y. Tachikawa, Supersymmetric Completion of an $R^{2}$ term in Five-dimensional Supergravity, Prog. Theor. Phys. 117 (2007) 533 [hep-th/0611329] [INSPIRE].

[45] R.E. Borcherds, Automorphic forms with singularities on Grassmannians, Invent. Math. 132 (1998) 491 [alg-geom/9609022].

[46] M. Mariño and G.W. Moore, Counting higher genus curves in a Calabi-Yau manifold, Nucl. Phys. B 543 (1999) 592 [hep-th/9808131] [INSPIRE]. 
[47] L.J. Dixon, V. Kaplunovsky and J. Louis, Moduli dependence of string loop corrections to gauge coupling constants, Nucl. Phys. B 355 (1991) 649 [INSPIRE].

[48] J.A. Harvey and G.W. Moore, Algebras, BPS states and strings, Nucl. Phys. B 463 (1996) 315 [hep-th/9510182] [INSPIRE].

[49] K. Foerger and S. Stieberger, Higher derivative couplings and heterotic type-I duality in eight-dimensions, Nucl. Phys. B 559 (1999) 277 [hep-th/9901020] [INSPIRE].

[50] K. Forger and S. Stieberger, String amplitudes and $N=2, D=4$ prepotential in heterotic K3 $3 T^{2}$ compactifications, Nucl. Phys. B 514 (1998) 135 [hep-th/9709004] [INSPIRE].

[51] W. Lerche and S. Stieberger, 1/4 BPS states and nonperturbative couplings in $N=4$ string theories, Adv. Theor. Math. Phys. 3 (1999) 1539 [hep-th/9907133] [INSPIRE].

[52] C. Angelantonj, I. Florakis and B. Pioline, A new look at one-loop integrals in string theory, Commun. Num. Theor. Phys. 6 (2012) 159 [arXiv:1110.5318] [InSPIRE].

[53] C. Angelantonj, I. Florakis and B. Pioline, One-Loop BPS amplitudes as BPS-state sums, JHEP 06 (2012) 070 [arXiv:1203.0566] [INSPIRE]. 